TRANSACTIONS OF THE

AMERICAN MATHEMATICAL SOCIETY

Volume 365, Number 11, November 2013, Pages 5805-5832

S 0002-9947(2013)05784-4

Article electronically published on July 10, 2013

\title{
FRACTIONAL ITERATION OF SERIES AND TRANSSERIES
}

\author{
G. A. EDGAR
}

\begin{abstract}
We investigate compositional iteration of fractional order for transseries. For any large positive transseries $T$ of exponentiality 0 , there is a family $T^{[s]}$ indexed by real numbers $s$ corresponding to iteration of order $s$. It is based on Abel's Equation. We also investigate the question of whether there is a family $T^{[s]}$ all sharing a single support set. A subset of the transseries of exponentiality 0 is divided into three classes ("shallow", "moderate" and "deep") with different properties related to fractional iteration.
\end{abstract}

\section{INTRODUCTION}

Since at least as long ago as 1860 (A. Cayley [4) there has been discussion of real iteration groups (compositional iteration of fractional order) for power series, or at least for formal power series, where we do not worry about convergence of the result. In this paper we adapt this to transseries. In many cases it is, in fact, not difficult to do when we ignore questions of convergence.

We will primarily use the ordered differential field $\mathbb{T}=\mathbb{R} \llbracket \mathfrak{G} \rrbracket=\mathbb{R} \mathbb{\mathbb { L }} x$ of (real grid-based) transseries; $\mathbb{T}$ is also known as the transline. So $\mathbb{T}$ is the set of all grid-based real formal linear combinations of monomials from $\mathfrak{G}$, while $\mathfrak{G}$ is the set of all $e^{L}$ for $L \in \mathbb{T}$ purely large. (Because of logarithms, there is no need to write two factors separately as $x^{b} e^{L}$.) See the "Review" below.

The problem looks like this: Let $T$ be a large positive transseries. Is there a family $T^{[s]}$ of transseries, indexed by reals $s$, so that $T^{[0]}(x)=x, T^{[1]}=T$, and $T^{[s]} \circ T^{[t]}=T^{[s+t]}$ for all $s, t \in \mathbb{R}$ ? These would be called fractional iterates of $T$ : $U=T^{[1 / 2]}$ satisfies $U \circ U=T$; or $T^{[-1]}$ is the compositional inverse of $T$; etc. We limit this discussion to large positive transseries since that is where compositions $S \circ T$ are always defined. In Corollary 4.5 we conclude (in the well-based case) that any large positive $T$ of exponentiality 0 admits such a family of fractional iterates. However, there are grid-based large positive transseries $T$ and reals $s$ for which the fractional iterate $T^{[s]}$ is not grid-based (Example 4.8).

We also investigate the existence of a family $T^{[s]}, s \in \mathbb{R}$, all supported by a single grid (in the grid-based case) or by a single well-ordered set (in the well-based case). We show that such a family exists in certain cases (Theorems 3.7 and 3.8) but not in other cases (Theorem 3.9).

The author thanks the referee for a careful reading of the manuscript, and for remarks, terminologies, and references.

Received by the editors February 25, 2010 and, in revised form, December 29, 2011.

2010 Mathematics Subject Classification. Primary 03C64; Secondary 41A60, 39B12, 30B10. 
Review. The differential field $\mathbb{T}$ of transseries is completely explained in the recent expository introduction [11. Other sources for the definitions are [1, [6, 9], 17. We will generally follow the notation from [11. The well-based version of the construction is described in 9] (or [12, Def. 2.1]). The term "hereditarily finite type" is used for "grid-based" when that setting is explored in [9, Sec. 7]. In this paper it is intended that all results hold for both versions, unless otherwise noted. (We use the labels $\mathrm{G}$ or $\mathrm{W}$ for statements or proofs valid only for the grid-based or well-based version, respectively.) We will write $\mathbb{T}, \mathfrak{G}$, and so on in both versions.

Write $\mathcal{P}=\{S \in \mathbb{T}: S \succ 1, S>0\}$ for the set of large positive transseries. The operation of composition $T \circ S$ is defined for $T \in \mathbb{T}, S \in \mathcal{P}$. The set $\mathcal{P}$ is a group under composition $([17, \S 5.4 .1]$ [9, Cor. 6.25] [12, Prop. 4.20] [13, Sec. 8]). Both notation $T \circ S$ and $T(S)$ will be used.

We write $\mathfrak{G}$ for the ordered abelian group of transmonomials. We write $\mathfrak{G}_{N, M}$ for the transmonomials with exponential height $N$ and logarithmic depth $M$. We write $\mathfrak{G}_{N}$ for the log-free transmonomials with height $N$. Even in the well-based case, the definition is restricted so that for any $T \in \mathbb{T}$ there exists $N, M$ with $\operatorname{supp} T \subseteq \mathfrak{G}_{N, M}$. The support $\operatorname{supp} T$ is well ordered for the converse of the relation $\prec$ in the wellbased case; the support $\operatorname{supp} T$ is a subgrid in the grid-based case. A ratio set $\boldsymbol{\mu}$ is a finite subset of $\mathfrak{G}^{\text {small }} ; \mathfrak{J}^{\boldsymbol{\mu}}$ is the group generated by $\boldsymbol{\mu}$. If $\boldsymbol{\mu}=\left\{\mu_{1}, \cdots, \mu_{n}\right\}$, then $\mathfrak{J}^{\mu}=\left\{\boldsymbol{\mu}^{\mathbf{k}}: \mathbf{k} \in \mathbb{Z}^{n}\right\}$. If $\mathbf{m} \in \mathbb{Z}^{n}$, then $\mathfrak{J}^{\boldsymbol{\mu}, \mathbf{m}}=\left\{\boldsymbol{\mu}^{\mathbf{k}}: \mathbf{k} \in \mathbb{Z}^{n}, \mathbf{k} \geq \mathbf{m}\right\}$ is a grid. A grid-based transseries is supported by some grid. A subgrid is a subset of a grid. (The referee remarks: Grid-based transseries are the ones to which, if even only hypothetically, one could ascribe analytic meaning.)

For transseries $A$, we already use exponents $A^{n}$ for multiplicative powers, and parentheses $A^{(n)}$ for derivatives. Therefore let us use square brackets $A^{[n]}$ for compositional powers. In particular, we will write $A^{[-1]}$ for the compositional inverse. Thus, for example, $\exp _{n}=\exp ^{[n]}=\log ^{[-n]}$.

Results from another paper. In this paper we will be using some of the notation and results on composition of transseries from [12, where the proofs are sometimes not as simple as in 11. We will use the following in particular: If $A, B \in \mathbb{T}$ and $A^{\prime}=B$, then we may write $A=\int B$. We also write $\int_{S_{1}}^{S_{2}} B:=A\left(S_{2}\right)-A\left(S_{1}\right)$ for $S_{1}, S_{2} \in \mathcal{P}, S_{1}<S_{2}$.

Proposition 0.1 ([12, Prop. 4.9]). Let $T \in \mathbb{T}, S_{1}, S_{2} \in \mathcal{P}$. If $T^{\prime}>0$ and $S_{1}<S_{2}$, then $T\left(S_{1}\right)<T\left(S_{2}\right)$.

Proposition 0.2 ([12, Prop. 4.10]). Let $A, B \in \mathbb{T}, S_{1}, S_{2} \in \mathcal{P}, S_{1}<S_{2}$. If $A^{\prime} \prec B^{\prime}$, then $A\left(S_{2}\right)-A\left(S_{1}\right) \prec B\left(S_{2}\right)-B\left(S_{1}\right)$.

The crucial and seemingly elementary facts of Propositions 0.1 and 0.2 are proved in [12, Sec. 8]. But - at least currently - the proofs are not simple.

Proposition 0.3 ([12, 4.17 and 4.18]). Let $A, B \in \mathbb{T}, S_{1}, S_{2} \in \mathcal{P}, S_{1}<S_{2}$. Then: $A>B \Longrightarrow \int_{S_{1}}^{S_{2}} A>\int_{S_{1}}^{S_{2}} B ; A \succ B \Longrightarrow \int_{S_{1}}^{S_{2}} A \succ \int_{S_{1}}^{S_{2}} B ; A \sim B \Longrightarrow \int_{S_{1}}^{S_{2}} A \sim \int_{S_{1}}^{S_{2}} B$.

The reformulations of Proposition 0.3 is so innocuous-looking that we may use them without noticing it.

Proposition 0.4 ([12, Prop. 4.23]). Given $A \in \mathbb{T}, S_{1}, S_{2} \in \mathcal{P}, S_{1}<S_{2}$, there is $S \in \mathcal{P}$ so that $\left(A\left(S_{2}\right)-A\left(S_{1}\right)\right) /\left(S_{2}-S_{1}\right)=A^{\prime}(S)$. 
The Mean Value Theorem 0.4 is proved in [12, based on Proposition 0.1 ,

Proposition 0.5. Given $A \in \mathbb{T}, S_{1}, S_{2} \in \mathcal{P}, S_{1}<S_{2}$, there is $S \in \mathcal{P}$ so that $S_{1}<S<S_{2}$ and $\left(A\left(S_{2}\right)-A\left(S_{1}\right)\right) /\left(S_{2}-S_{1}\right)=A^{\prime}(S)$.

The Mean Value Theorem[0.5] is stated as [12, Prop. 4.24] but not proved there. Related results are [17, Prop. 5.11(c)], 99, Thm. 6.2], 12, Prop. 7.1]. An alternate statement $\left(A\left(S_{2}\right)-A\left(S_{1}\right)\right) /\left(S_{2}-S_{1}\right)$ is between $A^{\prime}\left(S_{1}\right)$ and $A^{\prime}\left(S_{2}\right)$.

Unfortunately, some of the proofs in the present paper use Proposition 0.5 . So we need a proof for it. In fact, here we need only the special case: if $A^{\prime}\left(S_{1}\right) \sim$ $A^{\prime}\left(S_{2}\right)$, then $\left(A\left(S_{2}\right)-A\left(S_{1}\right)\right) /\left(S_{2}-S_{1}\right) \sim A^{\prime}\left(S_{1}\right)$, or: if $B\left(S_{1}\right) \sim B\left(S_{2}\right)$, then $\int_{S_{1}}^{S_{2}} B \sim B\left(S_{1}\right) \cdot\left(S_{2}-S_{1}\right)$.

\section{Motivation FOR FRACTIONAL ITERATiON}

Before we turn to transseries, let us consider fractional iteration in general. Given functions $T: X \rightarrow X$ and $\Phi: \mathbb{R} \times X \rightarrow X$, we say that $\Phi$ is a real iteration group for $T$ iff

$$
\begin{aligned}
\Phi(s+t, x) & =\Phi(s, \Phi(t, x)) \\
\Phi(0, x) & =x \\
\Phi(1, x) & =T(x)
\end{aligned}
$$

for all $s, t \in \mathbb{R}$ and $x \in X$.

Let us assume, say, that $X$ is an interval $(a, b) \subseteq \mathbb{R}$, possibly $a=-\infty$ and/or $b=\infty$, and that $\Phi$ has as many derivatives as needed. If we start with (1), take the partial derivative with respect to $t$,

$$
\Phi_{1}(s+t, x)=\Phi_{2}(s, \Phi(t, x)) \Phi_{1}(t, x),
$$

and then substitute $t=0$, to get

$$
\Phi_{1}(s, x)=\Phi_{2}(s, x) \Phi_{1}(0, x) .
$$

We have written $\Phi_{1}$ and $\Phi_{2}$ for the two partial derivatives of $\Phi$. Equation (4) is the one we will be using in Sections 2 and 3 below. It is known in iteration theory as the Aczél-Jabotinsky Equation; see [20, 21].

Here is a proof showing how it works in the case of functions on an interval $X$.

Proposition 1.1. Suppose $\Phi: \mathbb{R} \times X \rightarrow X$ satisfies (2) and

$$
\Phi_{1}(s, x)=\Phi_{2}(s, x) \beta(x)
$$

with $\beta(x)>0$. [Assume also $\infty=\int_{x_{0}}^{b} d y / \beta(y)$ and $\infty=\int_{a}^{x_{0}} d y / \beta(y)$ for $x_{0} \in X=$ $(a, b)$.] Then $\Phi$ satisfies (11) with $\beta(x)=\Phi_{1}(0, x)$.

Proof. Fix $x_{0} \in X$. Let $\theta(t)$ be the solution of the ODE $\theta^{\prime}(t)=\beta(\theta(t)), \theta(0)=x_{0}$. That is, $\theta(t)=x$ is defined implicitly by

$$
\int_{x_{0}}^{x} \frac{d y}{\beta(y)}=t
$$

[In order to get all time $t$, we need $\infty=\int_{x_{0}}^{b} d y / \beta(y)$ and $\infty=\int_{a}^{x_{0}} d y / \beta(y)$.] Consider $F(u, v)=\Phi(u+v, \theta(u-v))$. Then

$$
\begin{aligned}
F_{2}(u, v) & =\Phi_{1}(u+v, \theta(u-v))-\Phi_{2}(u+v, \theta(u-v)) \theta^{\prime}(u-v) \\
& =\Phi_{1}(u+v, \theta(u-v))-\Phi_{2}(u+v, \theta(u-v)) \beta(\theta(u-v))=0
\end{aligned}
$$


by (4). This means $F$ is independent of $v$. So

$$
\begin{aligned}
\theta(t) & =\Phi(0, \theta(t))=F(t / 2,-t / 2)=F(t / 2, t / 2) \\
& =\Phi(t, \theta(0))=\Phi\left(t, x_{0}\right), \\
\Phi\left(s, \Phi\left(t, x_{0}\right)\right) & =\Phi(s, \theta(t))=F((s+t) / 2,(s-t) / 2) \\
& =F((s+t) / 2,(s+t) / 2)=\Phi(s+t, \theta(0))=\Phi\left(s+t, x_{0}\right) .
\end{aligned}
$$

Differentiate $\Phi\left(t, x_{0}\right)=\theta(t)$ to get $\Phi_{1}\left(t, x_{0}\right)=\theta^{\prime}(t)=\beta(\theta(t))$, then substitute $t=0$ to get $\Phi_{1}\left(0, x_{0}\right)=\beta(\theta(0))=\beta\left(x_{0}\right)$.

\section{THREE EXAMPLES}

Power series. We start with the classical case of power series. (A. Cayley 1860 [4]; A. Korkine 1882 [19].) We will think of formal power series (for $x \rightarrow \infty$ ), and not actual functions. Consider a series of the form

$$
\begin{aligned}
T(x) & =x\left(1+\sum_{j=1}^{\infty} c_{j} x^{-j}\right) \\
& =x+\sum_{j=1}^{\infty} c_{j} x^{-j+1}=x+c_{1}+c_{2} x^{-1}+c_{3} x^{-2}+\cdots
\end{aligned}
$$

Such a series admits an iteration group of the same form. That is,

$$
\Phi(s, x)=x\left(1+\sum_{j=1}^{\infty} \alpha_{j}(s) x^{-j}\right) .
$$

In fact, $\alpha_{j}(s)$ is $s c_{j}+$ \{polynomial in $s, c_{1}, c_{2}, \ldots, c_{j-1}$ with rational coefficients, of degree $j-1$ in $s\}$. The first few terms are

$$
\begin{aligned}
\Phi(s, x) & =x+s c_{1}+s c_{2} x^{-1}+\left(s c_{3}+\frac{s(1-s)}{2} c_{1} c_{2}\right) x^{-2} \\
& +\left(s c_{4}+\frac{s(1-s)}{2}\left(2 c_{1} c_{3}+c_{2}^{2}\right)+\frac{s(1-s)(1-2 s)}{6} c_{1}^{2} c_{2}\right) x^{-3}+\cdots .
\end{aligned}
$$

Theorem 2.1. Let $T(x)$ be the power series (5). Define $\alpha_{j}: \mathbb{R} \rightarrow \mathbb{R}$ recursively by

$$
\begin{aligned}
\alpha_{1}(s)=s c_{1} & \\
\alpha_{j}(s)=s & \left(c_{j}-\int_{0}^{1} \sum_{j_{1}+j_{2}=j}\left(-j_{1}+1\right) \alpha_{j_{1}}(u) \alpha_{j_{2}}^{\prime}(0) d u\right) \\
& +\int_{0}^{s} \sum_{j_{1}+j_{2}=j}\left(-j_{1}+1\right) \alpha_{j_{1}}(u) \alpha_{j_{2}}^{\prime}(0) d u .
\end{aligned}
$$

Then the series $\Phi$ defined formally by (6) is a real iteration group for $T$.

Proof. Check (2), (3), (41).

Remark 2.2. The formulas are obtained by plugging (6) into the Aczél-Jabotinsky Equation (4), equating coefficients, and then integrating the resulting ODEs. Consequently, this is the unique solution of the form (6), at least with differentiable coefficients. 
Remark 2.3. Of course there is a corresponding formulation for series of the form

$$
T(z)=z\left(1+\sum_{j=1}^{\infty} c_{j} z^{j}\right)=z+c_{1} z^{2}+c_{2} z^{3}+\cdots
$$

Then we get $\Phi(s, z)=z\left(1+\sum_{j=1}^{\infty} \alpha_{j}(s) z^{j}\right)$, with

$$
\begin{aligned}
\alpha_{1}(s)= & s c_{1}, \\
\alpha_{j}(s)=s & \left(c_{j}-\int_{0}^{1} \sum_{j_{1}+j_{2}=j}\left(j_{1}+1\right) \alpha_{j_{1}}(u) \alpha_{j_{2}}^{\prime}(0) d u\right) \\
& +\int_{0}^{s} \sum_{j_{1}+j_{2}=j}\left(j_{1}+1\right) \alpha_{j_{1}}(u) \alpha_{j_{2}}^{\prime}(0) d u .
\end{aligned}
$$

The first few terms are

$$
\begin{aligned}
\Phi(s, z)=z & +s c_{1} z^{2}+\left(s c_{2}+s(s-1) c_{1}^{2}\right) z^{3} \\
& +\left(s c_{3}+\frac{5 s(s-1)}{2} c_{1} c_{2}+\frac{s(s-1)(2 s-3)}{2} c_{1}^{3}\right) z^{4}+\cdots
\end{aligned}
$$

Convergence. Here we have considered the formal series. Even if (5) converges for all $x$ (except 0), it need not follow that (6) converges. Indeed, one of the criticisms of Cayley [4] and Korkine [19] was that convergence was not proved. Baker [2] provides examples where a power series converges, but none of its noninteger iterates converge. Erdös and Jabotinsky 15] investigate the set of $s$ for which the series converges. By [3], [10], 23], there are only three possibilities: (A) the series (6) diverges for every $x \neq 0$ and $s \neq 0$; (B) there is some non-zero $s_{1}$ such that given $s$, (6) converges for sufficiently large $x$ if and only if $s / s_{1} \in \mathbb{Z}$; or (C) for every $s$, the series (6) converges for all sufficiently large $x$. (Thanks to the referee for this remark.)

Transseries, height and depth 0 . Let $B \subseteq(0, \infty)$ be a well ordered set (under the usual order). The transseries

$$
T(x)=x\left(1+\sum_{b \in B} c_{b} x^{-b}\right)=x+\sum_{b \in B} c_{b} x^{-b+1}
$$

is the next one we consider. In fact, the additive semigroup generated by $B$ is again well ordered (Higman; see the proof in [12, Prop. 2.2]), so we will assume from the start that $B$ is a semigroup. If $B$ is finitely generated, then (77) is a grid-based transseries. But in general it is well-based. If $B$ is finitely generated, then $B$ has order type $\omega$. But of course a well ordered $B$ can have an arbitrarily large countable ordinal as its order type.

We claim $T$ has a real iteration group supported by $1-B$, where $B$ is a well ordered additive semigroup of positive reals. 
Proposition 2.4. Let $B \subseteq(0, \infty)$ be a well ordered semigroup. The transseries (17) has a real iteration group

$$
\Phi(s, x)=x\left(1+\sum_{b \in B} \alpha_{b}(s) x^{-b}\right) .
$$

Proof. The only thing needed is that $B$ is a well ordered semigroup. It follows that, for any given $b \in B$, there are just finitely many pairs $\left(b_{1}, b_{2}\right) \in B \times B$ with $b_{1}+b_{2}=b$ [11, Prop. 3.27]. Then define recursively:

$$
\begin{aligned}
f_{b}(s) & =\sum_{b_{1}+b_{2}=b}\left(-b_{1}+1\right) \alpha_{b_{1}}(s) \alpha_{b_{2}}^{\prime}(0), \\
\alpha_{b}(s) & =s\left(c_{b}-\int_{0}^{1} f_{b}(u) d u\right)+\int_{0}^{s} f_{b}(u) d u .
\end{aligned}
$$

For each $b$, both $f_{b}(s)$ and $\alpha_{b}(s)$ are polynomials (finitely many terms!) in $s$ and $c_{b_{1}}$ [with $b_{1}<b$ except for the term $s c_{b}$ ]. Check (2), (3), (4).

A moderate example. Now we consider another case. We single it out because it occurs frequently enough to make it useful to have the formulas displayed. Consider the transseries

$$
T(x)=x\left(\sum_{k=0}^{\infty} \sum_{j=0}^{\infty} c_{j, k} x^{-j} e^{-k x}\right), \quad c_{0,0}=1 .
$$

The set

$$
B=\{(j, k) \in \mathbb{Z} \times \mathbb{Z}: k \geq 0, j \geq 0,(j, k) \neq(0,0)\}
$$

is a semigroup under addition. The set $\left\{x^{-j} e^{-k x}:(j, k) \in B\right\}$ is then a semigroup under multiplication. It is well ordered with order type $\omega^{2}$ with respect to the converse of $\succ$.

Theorem 2.5. Let $B$ be as in (9). Then the transseries (8) admits a real iteration group supported by the same set $\left\{x^{1-j} e^{-k x}: j, k \geq 0\right\}$.

Proof. Write

$$
\Phi(s, x)=x\left(1+\sum_{(j, k) \in B} \alpha_{j, k}(s) x^{-j} e^{-k x}\right) .
$$

(A) We first consider the case with $c_{1,0}=0$. The coefficient functions $\alpha_{j, k}$ are defined recursively as follows. If $(j, k) \notin B$, then let $\alpha_{j, k}(s)=0$. Let $(j, k) \in B$ and assume $\alpha_{j_{1}, k_{1}}(s)$ have already been defined for all $\left(j_{1}, k_{1}\right)$ with either $k_{1}<k$ or $\left\{k_{1}=k\right.$ and $\left.j_{1}<j\right\}$. Then let

$$
f_{j, k}(s)=\sum\left[\left(-j_{1}+1\right) \alpha_{j_{2}, k_{2}}^{\prime}(0)-k_{1} \alpha_{j_{2}+1, k_{2}}^{\prime}(0)\right] \alpha_{j_{1}, k_{1}}(s),
$$

where the sum is over all $j_{1}, j_{2}, k_{1}, k_{2}$ with $j_{1}+j_{2}=j, k_{1}+k_{2}=k$. Check that all the terms in the sum involve $\alpha$ 's that have already been defined (or are multiplied by zero); this depends on $c_{1,0}=0$, so $\alpha_{1,0}(s)=0$. Define $F_{j, k}(s)=\int_{0}^{s} f_{j, k}(u) d u$ and

$$
\alpha_{j, k}(s)=\left(c_{j, k}-F_{j, k}(1)\right) s+F_{j, k}(s)
$$

Check (2), (3), (4). 
(B) Now consider the case with $c_{1,0} \neq 0$. Write $c=c_{1,0}$. The coefficient functions $\alpha_{j, k}$ are defined recursively as follows. If $(j, k) \notin B$, then let $\alpha_{j, k}(s)=0$. Let $(j, k) \in B$ and assume $\alpha_{j_{1}, k_{1}}(s)$ have already been defined for all $\left(j_{1}, k_{1}\right)$ with either $k_{1}<k$ or $\left\{k_{1}=k\right.$ and $\left.j_{1}<j\right\}$. Then let

$$
f_{j, k}(s)=\sum\left[\left(-j_{1}+1\right) \alpha_{j_{2}, k_{2}}^{\prime}(0)-k_{1} \alpha_{j_{2}+1, k_{2}}^{\prime}(0)\right] \alpha_{j_{1}, k_{1}}(s),
$$

where the sum is over all $j_{1}, j_{2}, k_{1}, k_{2}$ with $j_{1}+j_{2}=j, k_{1}+k_{2}=k$. Omit the terms $\alpha_{j k}^{\prime}(0)$ and $-k c \alpha_{j k}(s)$ and terms with a factor 0 . Then all terms in the sum involve $\alpha$ 's already defined. Define $F_{j, k}(s)=\int_{0}^{s} e^{k c(u-s)} f_{j, k}(u) d u$ and

$$
\alpha_{j, k}(s)= \begin{cases}\left(c_{j, 0}-F_{j, 0}(1)\right) s+F_{j, 0}(s), & \text { if } k=0, \\ \left(c_{j, k}-F_{j, k}(1)\right) \frac{1-e^{-s c k}}{1-e^{-c k}}+F_{j, k}(s), & \text { if } k>0 .\end{cases}
$$

Recall $c=c_{1,0}$. Check (2), (3), (4)).

In case (B) - the "moderate case" - the coefficients $\alpha_{j, k}(s)$ are not necessarily polynomials in $s$. The referee pointed out that this is a genuinely new phenomenon in transseries, which does not occur in the context of formal power series.

A deep example. Another simple example shows that a real iteration group of that type need not always exist. Let $B \subseteq \mathbb{Z}^{3}$ be

$$
B=\{(j, 0,0): j \geq 1\} \cup\{(j, k, 0): k \geq 1\} \cup\{(j, k, l): l \geq 1\} .
$$

Then $\left\{x^{-j} e^{-k x} e^{-l x^{2}}:(j, k, l) \in B\right\}$ is a semigroup, but not well ordered.

We included some negative $j$ and $k$ so that the set of transseries of the form

$$
x\left(1+\sum_{(j, k, l) \in B} c_{j k l} x^{-j} e^{-k x} e^{-l x^{2}}\right)
$$

(where of course each individual support is well ordered, not all of $B$ ) is closed under composition.

Proposition 2.6. Let $B^{\prime}$ be a well ordered subset of (10). The transseries $T(x)=$ $x\left(1+x^{-1}+e^{-x^{2}}\right)$ admits no real iteration group of the form

$$
\Phi(s, x)=x\left(1+\sum_{(j, k, l) \in B^{\prime}} \alpha_{j k l}(s) x^{-j} e^{-k x} e^{-l x^{2}}\right) .
$$

Proof. We may assume $B^{\prime}=\left\{(j, k, l): \alpha_{j k l} \neq 0\right\}$. As before, the first term beyond $x$ can be computed as $\alpha_{100}(s)=s \cdot 1=s$, so $\alpha_{100}^{\prime}(s)=1$. Now $\alpha_{001}(1) \neq 0$, so there is a least $(j, k, 1) \in B^{\prime}$. But then by considering the coefficient of $x^{2-j} e^{-k x} e^{-x^{2}}$ in the Aczél-Jabotinsky Equation $\Phi_{1}(s, x)=\Phi_{2}(s, x) \Phi_{1}(0, x)$ we have

$$
0=(-2) \alpha_{j k 1}(s) \alpha_{100}^{\prime}(0),
$$

so $\alpha_{j k 1}(s)=0$, a contradiction. 
Remark 2.7. Here is an alternate argument is that there is no grid (or well ordered set) supporting a real iteration group for $T=x+1+x e^{-x^{2}}$. Compute

$$
\begin{aligned}
\operatorname{supp} T^{[-1]} & =\left\{x \succ 1 \succ x e^{2 x} e^{-x^{2}} \succ \cdots\right\}, \\
\operatorname{supp} T^{[-2]} & =\left\{x \succ 1 \succ x e^{4 x} e^{-x^{2}} \succ \cdots\right\}, \\
\operatorname{supp} T^{[-3]} & =\left\{x \succ 1 \succ x e^{6 x} e^{-x^{2}} \succ \cdots\right\} \\
& \cdots \\
\operatorname{supp} T^{[-k]} & =\left\{x \succ 1 \succ x e^{2 k x} e^{-x^{2}} \succ \cdots\right\}, \quad k \in \mathbb{N}, k>0 .
\end{aligned}
$$

So there is no grid (and no well ordered set) containing all of these supports.

\section{The CASE OF COMMON SUPPORT}

Conjugation. The set $\mathcal{P}$ of large positive transseries is a group under composition ([17, $\S 5.4 .1]$, 9, Cor. 6.25], [12, Prop. 4.20], [13, Sec. 8]). We say $U, V$ are conjugate if there exists $S \in \mathcal{P}$ with $S^{[-1]} \circ U \circ S=V$. Then for all $k \in \mathbb{N}$ it follows that $S^{[-1]} \circ U^{[k]} \circ S=V^{[k]}$. If $\Phi(s, x)$ is an iteration group for $U(x)$, then $S^{[-1]}(\Phi(s, S(x)))$ is an iteration group for the conjugate $S^{[-1]}(U(S(x)))$. This can be used to reduce the question of fractional iteration for certain more general transseries to more restricted cases that will be discussed here.

Puiseux series. For a Puiseux series of the form

$$
T=\sum_{j=m}^{\infty} c_{j} x^{-j / k}
$$

$\left(m \in \mathbb{Z}, k \in \mathbb{N}, c_{m}>0\right)$, we can conjugate with $x^{1 / k}$ :

$$
\begin{aligned}
x^{1 / k} \circ T \circ x^{k} & =\left(T\left(x^{k}\right)\right)^{1 / k}=\left(\sum_{j=m}^{\infty} c_{j} x^{-j}\right)^{1 / k} \\
& =c_{m}^{1 / k} x^{-m / k}\left(1+c_{m+1} x^{-1}+c_{m+2} x^{-2}+\cdots\right)^{1 / k} \\
& =c_{m}^{1 / k} x^{-m / k}\left(1+a_{1} x^{-1}+a_{2} x^{-2}+\cdots\right) .
\end{aligned}
$$

If $\operatorname{dom} T=x$, then also $\operatorname{dom}\left(x^{1 / k} \circ T \circ x^{k}\right)=x$, and existence of a real iteration group is then clear from Proposition 2.1. (If $\operatorname{dom} T \neq x$, keep reading.)

Exponentiality. Associated to a general $T \in \mathcal{P}$ is an integer $p$ called the exponentiality of $T$ [17, Ex. 4.10], [12, Prop. 4.5] such that for all large enough $k \in \mathbb{N}$ we have $\log _{k} \circ T \circ \exp _{k} \sim \exp _{p}$. Write $p=\operatorname{expo} T$. [The exponentiality of $T$ is called the level of $T$ in [9], employing terminology introduced in [24] in the context of Hardy fields.]

Now $\operatorname{expo}(S \circ T)=\operatorname{expo} S+\operatorname{expo} T$, so no transseries with non-zero exponentiality can have a real iteration group of transseries. In particular, there is no transseries $T$ with $T \circ T=e^{x}$. (But see [14].) The main question will be for exponentiality zero. If expo $T=0$, then $T$ is conjugate to some $S=\log _{k} \circ T \circ \exp _{k}$ such that $S \sim x$ and such that $S$ is log-free [12, Prop. 4.8]. So we will deal with this case. 
Shallow - moderate - deep. Now we turn to the general large positive log-free transseries with dominant term $x$. It admits a unique real iteration group with a common support in many cases (shallow and moderate), but not in many other cases (deep).

Definition 3.1. Consider log-free $T \sim x$. A real iteration group for $T$ with common support is a real iteration group $\Phi(s, x)$ of the form

$$
\Phi(s, x)=x\left(1+\sum_{\mathfrak{g} \in \mathfrak{B}} \alpha_{\mathfrak{g}}(s) \mathfrak{g}\right)
$$

for some subgrid ( $\mathrm{W}$ or well ordered) $\mathfrak{B} \subseteq \mathfrak{G}$ (not depending on $s$ ) where coefficient functions $\alpha_{\mathfrak{g}}: \mathbb{R} \rightarrow \mathbb{R}$ are differentiable. (Recall that $\mathfrak{G}$ denotes the ordered group of all transmonomials.)

Write $T=x(1+U), U \prec 1, U \sim a \mathfrak{e}, a \in \mathbb{R}, a \neq 0, \mathfrak{e} \in \mathfrak{G}, \mathfrak{e} \prec 1$. As before, if there is a real interation group $\Phi$, it begins with $\Phi(s, x)=x(1+s a \mathfrak{e}+\cdots)$. We may assume that if $\mathfrak{g} \in \mathfrak{B}$, then $\alpha_{\mathfrak{g}}(s) \neq 0$ for some $s$. So the greatest element of $\mathfrak{B}$ is $\mathfrak{e}$.

Write $A^{\dagger}=A^{\prime} / A$ for the logarithmic derivative.

Definition 3.2. Let $T=x(1+U), U \prec 1, \operatorname{mag} U=\mathfrak{e}$. The monomial $\mathfrak{e}$ is called the first ratio of $T$. We say that $T$ is:

shallow iff $\mathfrak{g}^{\dagger} \prec 1 /(x \mathfrak{e})$ for all $\mathfrak{g} \in \operatorname{supp} U$;

moderate iff $\mathfrak{g}^{\dagger} \preccurlyeq 1 /(x \mathfrak{e})$ for all $\mathfrak{g} \in \operatorname{supp} U$ and $\mathfrak{g}^{\dagger} \asymp 1 /(x \mathfrak{e})$ for at least one $\mathfrak{g} \in \operatorname{supp} U$

deep iff $\mathfrak{g}^{\dagger} \succ 1 /(x \mathfrak{e})$ for some $\mathfrak{g} \in \operatorname{supp} U$;

purely deep iff $\mathfrak{g}^{\dagger} \succ 1 /(x \mathfrak{e})$ for all $\mathfrak{g} \in \operatorname{supp} U$ except $\mathfrak{e}$.

(In the case of ordinary power series, the monomial $\mathfrak{e}$ - or its multiplicative inverse - also plays an important role in the theory of conjugate power series. In Écalle's terminology for power series, $\mathfrak{e}$ is called the iterative valuation of $T$. See [21, pp. 346-364].)

Remark 3.3. G It may be practical to check these definitions by using a ratio set $\boldsymbol{\mu}=\left\{\mu_{1}, \cdots, \mu_{n}\right\}$. For example, suppose $\operatorname{supp} U \subseteq \mathfrak{J}^{\mu}$. By the group property (Lemma 3.14), if $\mu_{i}^{\dagger} \prec 1 /(x \mathfrak{e})$ for $1 \leq i \leq n$, then $T$ is shallow. This will be "if and only if" provided $\boldsymbol{\mu}$ is chosen from the group generated by $\operatorname{supp} U$, which can always be done.

Remark 3.4. The case of two terms, $T=x(1+a \mathfrak{e})$ exactly, is shallow. Indeed, $\mathfrak{e} \prec 1$, and so $x \mathfrak{e} \prec x, x \mathfrak{e}^{\prime} \prec 1$ and $\mathfrak{e}^{\prime} / \mathfrak{e} \prec 1 /(x \mathfrak{e})$.

Remark 3.5. For small monomials, the logarithmic derivative operation reverses the order: if $1 \succ \mathfrak{a} \succ \mathfrak{b}$, then $\mathfrak{a}^{\dagger} \preccurlyeq \mathfrak{b}^{\dagger}$ (Lemma 3.11(e)). Also, for $U \prec 1$ we have $U^{\dagger} \sim(\operatorname{mag} U)^{\dagger}($ Lemma 3.11(c)). So $T=x(1+a \mathfrak{e}+V), V \prec \mathfrak{e}$, is purely deep if and only if $V^{\dagger} \succ 1 /(x \mathfrak{e})$.

Remark 3.6. The condition $\mathfrak{g}^{\dagger} \prec 1 /(x \mathfrak{e})$ says that $\mathfrak{g}$ is "not too small" in relation to $\mathfrak{e}$. (This is the reason for the terms "shallow" and "deep".) If $\mathfrak{g} \prec 1$, then $\mathfrak{g}=e^{-L}$ 
with $L>0$ purely large and

$$
\begin{aligned}
\mathfrak{g}^{\dagger} \prec \frac{1}{x \mathfrak{e}} & \Longleftrightarrow L^{\prime} \prec \frac{1}{x \mathfrak{e}} \\
& \Longleftrightarrow L \prec \int \frac{1}{x \mathfrak{e}} \\
& \Longleftrightarrow L<c \int \frac{1}{x \mathfrak{e}} \text { for all real } c>0 \\
& \Longleftrightarrow e^{L}<\exp \left(c \int \frac{1}{x \mathfrak{e}}\right) \text { for all real } c>0 \\
& \Longleftrightarrow \mathfrak{g}>\exp \left(-c \int \frac{1}{x \mathfrak{e}}\right) \text { for all real } c>0
\end{aligned}
$$

So the set $\mathfrak{A}=\left\{\mathfrak{g} \in \mathfrak{G}: \mathfrak{g} \preccurlyeq \mathfrak{e}, \mathfrak{g}^{\dagger} \prec 1 /(x \mathfrak{e})\right\}$ is an interval in $\mathfrak{G}$. The large end of the interval is the first ratio $\mathfrak{e}$, and the small end of the interval is the gap in $\mathfrak{G}$ just above all the values $\exp \left(-c \int(1 /(x \mathfrak{e}))\right), c \in \mathbb{R}, c>0$. If we write $\exp \left(-\overline{0} \int(1 /(x \mathfrak{e}))\right)$ for that gap, then

$$
\left.\mathfrak{A}=] \exp \left(-\overline{0} \int(1 /(x \mathfrak{e}))\right), \mathfrak{e}\right]
$$

We will call this the shallow interval below $\mathfrak{e}$. Van der Hoeven devotes a chapter [17. Chap. 9] to gaps (cuts) in the transline. In his classification [17, Prop. 9.15],

$$
\exp \left(-\overline{0} \int(1 /(x \mathfrak{e}))\right)=e^{-e^{A-e^{\bar{m}}}},
$$

where $\operatorname{mag} \int(1 /(x \mathfrak{e}))=e^{A}$.

Similarly, the set $\mathfrak{A}=\left\{\mathfrak{g} \in \mathfrak{G}: \mathfrak{g} \preccurlyeq \mathfrak{e}, \mathfrak{g}^{\dagger} \preccurlyeq 1 /(x \mathfrak{e})\right\}$ is an interval in $\mathfrak{G}$. The large end of the interval is the first ratio $\mathfrak{e}$, and the small end of the interval is the gap in $\mathfrak{G}$ just below all the values $\exp \left(-c \int(1 /(x \mathfrak{e}))\right), c \in \mathbb{R}, c>0$. If we write $\exp \left(-\bar{\infty} \int(1 /(x \mathfrak{e}))\right)$ for that gap, then

$$
\left.\mathfrak{A}=] \exp \left(-\bar{\infty} \int(1 /(x \mathfrak{e}))\right), \mathfrak{e}\right] .
$$

We will call this the moderate interval below $\mathfrak{e}$. In van der Hoeven's classification,

$$
\exp \left(-\bar{\infty} \int(1 /(x \mathfrak{e}))\right)=e^{-e^{A+e^{\bar{m}}}}
$$

where $\operatorname{mag} \int(1 /(x \mathfrak{e}))=e^{A}$.

(We follow [17] in calling these sets "intervals" despite the fact that the lower "endpoint" is a gap and not an element of $\mathfrak{G}$. Would it perhaps be reasonable to refer to them simply as "convex sets"?)

The examples. Let us examine where the examples done above fit in the shallow/deep classification. If $\mathfrak{e}=x^{-1}$, then

$$
\frac{1}{x \mathfrak{e}}=1, \quad \int \frac{1}{x \mathfrak{e}}=x, \quad \exp \left(-c \int \frac{1}{x \mathfrak{e}}\right)=e^{-c x} .
$$

The small end of the shallow interval is $\exp (-\overline{0} x)$. In a power series, every monomial $x^{-j} \succ e^{-\overline{0} x}$ is inside the shallow interval, so a power series is shallow.

In Theorem 2.5. we saw two cases. In case $c_{1,0} \neq 0$, then $\mathfrak{e}=x^{-1}$, so again the small end of the shallow interval is $\exp (-\overline{0} x)$. But the monomial $x^{-j} e^{-k x} \prec$ $\exp (-\overline{0} x)$ if $k>0$, and is thus outside the shallow interval, so this is not shallow. The small end of the moderate interval is $\exp (-\bar{\infty} x)$, and all monomials $x^{-j} e^{-k x} \succ$ $\exp (-\bar{\infty} x)$ are inside the moderate interval, so this is the moderate case. 
The other case is $c_{1,0}=0$. Then $\mathfrak{e}$ is $x^{-2}$ (or smaller). If $\mathfrak{e}=x^{-2}$, then

$$
\frac{1}{x \mathfrak{e}}=x, \quad \int \frac{1}{x \mathfrak{e}}=\frac{x^{2}}{2}, \quad \exp \left(-c \int \frac{1}{x \mathfrak{e}}\right)=e^{-(c / 2) x^{2}} .
$$

The small end of the shallow interval is $\exp \left(-\overline{0} x^{2}\right)$. All monomials $x^{-j} e^{-k x} \succ$ $\exp \left(-\overline{0} x^{2}\right)$ are inside the shallow interval, so this is the shallow case.

Finally consider the example $T=x\left(1+x^{-1}+e^{-x^{2}}\right)$ of Proposition 2.6. Since $\mathfrak{e}=x^{-1}$, the small end of the moderate interval was computed as $\exp (-\bar{\infty} x)$. The monomial $e^{-x^{2}} \prec \exp (-\bar{\infty} x)$ is outside of that, so $T$ is deep.

Proofs. Proofs will follow the examples done above. These proofs use some technical lemmas on logarithmic derivatives, grids, and well ordered sets; they are found after the main results, starting with Lemma 3.11

Theorem 3.7. If (log-free) $T \sim x$ is shallow, then $T$ admits a real iteration group with common support where all coefficient functions are polynomials.

Proof. The proof is as in Proposition 2.4 above. Here are the details. Write $T=$ $x(1+U), U \sim a \mathfrak{e}, \mathfrak{e} \prec 1$. Begin with the subgrid ( $\mathrm{W}$ well ordered) $\operatorname{supp} U$ which is contained in $\left\{\mathfrak{g} \in \mathfrak{G}: \mathfrak{g} \preccurlyeq \mathfrak{e}, \mathfrak{g}^{\dagger} \prec 1 /(x \mathfrak{e})\right\}$. Let $\mathfrak{B} \supseteq \operatorname{supp} U$ be the least set such that if $\mathfrak{g}_{1}, \mathfrak{g}_{2} \in \mathfrak{B}$, then $\operatorname{supp}\left(\left(x \mathfrak{g}_{1}\right)^{\prime} \mathfrak{g}_{2}\right) \subseteq \mathfrak{B}$. By Lemma 3.22, $\mathfrak{B}$ is a subgrid $(W$ by Lemma 3.23, $\mathfrak{B}$ is well ordered) and $\mathfrak{B} \subseteq\left\{\mathfrak{g} \in \mathfrak{G}: \mathfrak{g} \preccurlyeq \mathfrak{e}, \mathfrak{g}^{\dagger} \prec 1 /(x \mathfrak{e})\right\}$.

Write

$$
\begin{aligned}
T(x) & =x\left(1+\sum_{\mathfrak{g} \in \mathfrak{B}} c_{\mathfrak{g}} \mathfrak{g}\right) \\
\Phi(s, x) & =x\left(1+\sum_{\mathfrak{g} \in \mathfrak{B}} \alpha_{\mathfrak{g}}(s) \mathfrak{g}\right) \\
\Phi_{1}(s, x) & =x \sum_{\mathfrak{g} \in \mathfrak{B}} \alpha_{\mathfrak{g}}^{\prime}(s) \mathfrak{g}, \\
\Phi_{1}(0, x) & =x \sum_{\mathfrak{g} \in \mathfrak{B}} \alpha_{\mathfrak{g}}^{\prime}(0) \mathfrak{g}, \\
\Phi_{2}(s, x) & =1+\sum_{\mathfrak{g} \in \mathfrak{B}} \alpha_{\mathfrak{g}}(s)(x \mathfrak{g})^{\prime}, \\
\Phi_{2}(s, x) \Phi_{1}(0, x) & =x \sum_{\mathfrak{g} \in \mathfrak{B}} \alpha_{\mathfrak{g}}^{\prime}(0) \mathfrak{g}+x \sum_{\mathfrak{g}_{1}, \mathfrak{g}_{2} \in \mathfrak{B}} \alpha_{\mathfrak{g}_{1}}(s) \alpha_{\mathfrak{g}_{2}}^{\prime}(0)\left(x \mathfrak{g}_{1}\right)^{\prime} \mathfrak{g}_{2} .
\end{aligned}
$$

Now fix a monomial $\mathfrak{g} \in \mathfrak{B}$. Assume $\alpha_{\mathfrak{g}_{1}}$ has been defined for all $\mathfrak{g}_{1} \succ \mathfrak{g}$. Consideration of the coefficient of $x \mathfrak{g}$ in the Aczél-Jabotinsky Equation (4) gives us an equation

$$
\alpha_{\mathfrak{g}}^{\prime}(s)=\alpha_{\mathfrak{g}}^{\prime}(0)+f_{\mathfrak{g}}(s),
$$

where $f_{\mathfrak{g}}(s)$ is a (real) linear combination of terms $\alpha_{\mathfrak{g}_{1}}(s) \alpha_{\mathfrak{g}_{2}}^{\prime}(0)$, where $\mathfrak{g}_{1}, \mathfrak{g}_{2} \in \mathfrak{B}$ satisfy $\mathfrak{g} \in \operatorname{supp}\left(\left(x \mathfrak{g}_{1}\right)^{\prime} \mathfrak{g}_{2}\right)$. By Lemma 3.19 ( $(\mathrm{W}$ Lemma 3.20$)$, for a given value of $\mathfrak{g}$, there are only finitely many pairs $\mathfrak{g}_{1}, \mathfrak{g}_{2}$ involved. 
Now we claim these all have $\mathfrak{g}_{1} \succ \mathfrak{g}$ and $\mathfrak{g}_{2} \succ \mathfrak{g}$. Indeed, since $\mathfrak{g} \in \operatorname{supp}\left(\left(x \mathfrak{g}_{1}\right)^{\prime} \mathfrak{g}_{2}\right)$ and $\left(x \mathfrak{g}_{1}\right)^{\prime}=\mathfrak{g}_{1}+x \mathfrak{g}_{1}^{\prime}$, we have either $\mathfrak{g} \preccurlyeq \mathfrak{g}_{1} \mathfrak{g}_{2}$ or $\mathfrak{g} \preccurlyeq x \mathfrak{g}_{1}^{\prime} \mathfrak{g}_{2}$. Take two cases:

(a) $\mathfrak{g} \preccurlyeq \mathfrak{g}_{1} \mathfrak{g}_{2}:$ Now $\mathfrak{g}_{1} \preccurlyeq \mathfrak{e}$, so $\mathfrak{g} \preccurlyeq \mathfrak{e} \mathfrak{g}_{2} \prec \mathfrak{g}_{2}$. Also, $\mathfrak{g}_{2} \preccurlyeq \mathfrak{e}$, so $\mathfrak{g} \preccurlyeq \mathfrak{e} \mathfrak{g}_{1} \prec \mathfrak{g}_{1}$.

(b) $\mathfrak{g} \preccurlyeq x \mathfrak{g}_{1}^{\prime} \mathfrak{g}_{2}:$ Now $\mathfrak{g}_{1}^{\prime} \prec \mathfrak{g}_{1} /(x \mathfrak{e})$, so $\mathfrak{g} \preccurlyeq x \mathfrak{g}_{1}^{\prime} \mathfrak{g}_{2} \prec \mathfrak{g}_{1} \mathfrak{g}_{2} / \mathfrak{e}$. But $\mathfrak{g}_{1} \preccurlyeq \mathfrak{e}$, so $\mathfrak{g} \prec \mathfrak{g}_{1}$, and $\mathfrak{g}_{2} \preccurlyeq \mathfrak{e}$, so $\mathfrak{g} \prec \mathfrak{g}_{2}$.

Thus we may use equation (14) to recursively define $\alpha_{\mathfrak{g}}(s)$. Indeed, solving the differential equation, we get $\alpha_{\mathfrak{g}}(s)=\int_{0}^{s} f_{\mathfrak{g}}(u) d u+s \alpha_{\mathfrak{g}}^{\prime}(0)+C$; but $\alpha_{\mathfrak{g}}(0)=0$ and $\alpha_{\mathfrak{g}}(1)=c_{\mathfrak{g}}$, so

$$
\alpha_{\mathfrak{g}}(s)=s\left(c_{\mathfrak{g}}-\int_{0}^{1} f_{\mathfrak{g}}(u) d u\right)+\int_{0}^{s} f_{\mathfrak{g}}(u) d u
$$

In particular, the recursion begins with $\mathfrak{g}=\mathfrak{e}$, where $f_{\mathfrak{e}}(s)=0$ and $\alpha_{\mathfrak{e}}(s)=s c_{\mathfrak{e}}=s a$. Also (by induction) $\alpha_{\mathfrak{g}}(s)$ and $f_{\mathfrak{g}}(s)$ are polynomials in $s$.

Theorem 3.8. If (log-free) $T \sim x$ is moderate, then $T$ admits a real iteration group with common support. The coefficient functions are entire; we cannot conclude that the coefficient functions are polynomials.

Proof. The proof is as in Theorem 2.5 above. Here are the details. Write $T=$ $x(1+U), U \sim a \mathfrak{e}, \mathfrak{e} \prec 1$. Begin with the subgrid ( $\mathrm{W}$ well ordered) $\operatorname{supp} U$ which is contained in $\left\{\mathfrak{g} \in \mathfrak{G}: \mathfrak{g} \preccurlyeq \mathfrak{e}, \mathfrak{g}^{\dagger} \preccurlyeq 1 /(x \mathfrak{e})\right\}$. Let $\mathfrak{B} \supseteq \operatorname{supp} U$ be the least set such that if $\mathfrak{g}_{1}, \mathfrak{g}_{2} \in \mathfrak{B}$, then $\operatorname{supp}\left(\left(x \mathfrak{g}_{1}\right)^{\prime} \mathfrak{g}_{2}\right) \subseteq \mathfrak{B}$. By Lemma 3.22, $\mathfrak{B}$ is a subgrid $(\mathrm{W}$ by Lemma 3.23, $\mathfrak{B}$ is well ordered) and $\mathfrak{B} \subseteq\left\{\mathfrak{g} \in \mathfrak{G}: \mathfrak{g} \preccurlyeq \mathfrak{e}, \mathfrak{g}^{\dagger} \preccurlyeq 1 /(x \mathfrak{e})\right\}$.

Write $T(x)=x\left(1+\sum_{\mathfrak{g} \in \mathfrak{B}} c_{\mathfrak{g}} \mathfrak{g}\right)$ and $\Phi(s, x)=x\left(1+\sum_{\mathfrak{g} \in \mathfrak{B}} \alpha_{\mathfrak{g}}(s) \mathfrak{g}\right)$. Compute the derivatives as in (13).

Now fix a monomial $\mathfrak{g} \in \mathfrak{B}$. Assume $\alpha_{\mathfrak{g}_{1}}$ has been defined for all $\mathfrak{g}_{1} \succ \mathfrak{g}$. There are two cases. If $\mathfrak{g}^{\dagger} \prec 1 /(x \mathfrak{e})$, then the argument proceeds as before, and we get (15). Now assume $\mathfrak{g}^{\dagger} \asymp 1 /(x \mathfrak{e})$, say $\mathfrak{g}^{\dagger} \sim b /(x \mathfrak{e}), b \in \mathbb{R}, b \neq 0$. Consideration of the coefficient of $x \mathfrak{g}$ in the Aczél-Jabotinsky Equation (4) gives us an ODE

$$
\alpha_{\mathfrak{g}}^{\prime}(s)=\alpha_{\mathfrak{g}}^{\prime}(0)+f_{\mathfrak{g}}(s)+b \alpha_{\mathfrak{g}}(s) \alpha_{\mathfrak{e}}^{\prime}(0),
$$

where $f_{\mathfrak{g}}(s)$ is a (real) linear combination of terms $\alpha_{\mathfrak{g}_{1}}(s) \alpha_{\mathfrak{g}_{2}}^{\prime}(0)$, where $\mathfrak{g}_{1}, \mathfrak{g}_{2} \in \mathfrak{B}$ satisfy $\mathfrak{g} \in \operatorname{supp}\left(\left(x \mathfrak{g}_{1}\right)^{\prime} \mathfrak{g}_{2}\right)$. The term with $\mathfrak{g}_{1}=\mathfrak{g}, \mathfrak{g}_{2}=\mathfrak{e}$, namely $b \alpha_{\mathfrak{g}}(s) \alpha_{\mathfrak{e}}^{\prime}(0)$, is not included in $f_{\mathfrak{g}}$ but written separately. By Lemma 3.19 ( $\mathrm{W}$ Lemma 3.20), for a given value of $\mathfrak{g}$, there are only finitely many pairs $\mathfrak{g}_{1}, \mathfrak{g}_{2}$ involved.

But we claim these all have $\mathfrak{g}_{1} \succ \mathfrak{g}$ and $\mathfrak{g}_{2} \succ \mathfrak{g}$ except for the case $\mathfrak{g}_{1}=\mathfrak{g}, \mathfrak{g}_{2}=\mathfrak{e}$. Indeed, since $\mathfrak{g} \in \operatorname{supp}\left(\left(x \mathfrak{g}_{1}\right)^{\prime} \mathfrak{g}_{2}\right)$ and $\left(x \mathfrak{g}_{1}\right)^{\prime}=\mathfrak{g}_{1}+x \mathfrak{g}_{1}^{\prime}$, we have either $\mathfrak{g} \preccurlyeq \mathfrak{g}_{1} \mathfrak{g}_{2}$ or $\mathfrak{g} \preccurlyeq x \mathfrak{g}_{1}^{\prime} \mathfrak{g}_{2}$. Take two cases:

(a) $\mathfrak{g} \preccurlyeq \mathfrak{g}_{1} \mathfrak{g}_{2}$ : Now $\mathfrak{g}_{1} \preccurlyeq \mathfrak{e}$, so $\mathfrak{g} \preccurlyeq \mathfrak{e g}_{2} \prec \mathfrak{g}_{2}$. Also, $\mathfrak{g}_{2} \preccurlyeq \mathfrak{e}$, so $\mathfrak{g} \preccurlyeq \mathfrak{e g}_{1} \prec \mathfrak{g}_{1}$.

(b) $\mathfrak{g} \preccurlyeq x \mathfrak{g}_{1}^{\prime} \mathfrak{g}_{2}$ : Now $\mathfrak{g}_{1}^{\prime} \preccurlyeq \mathfrak{g}_{1} /(x \mathfrak{e})$, so $\mathfrak{g} \preccurlyeq x \mathfrak{g}_{1}^{\prime} \mathfrak{g}_{2} \preccurlyeq \mathfrak{g}_{1} \mathfrak{g}_{2} / \mathfrak{e}$, with $\asymp$ only if $\mathfrak{g} \asymp x \mathfrak{g}_{1}^{\prime} \mathfrak{g}_{2}$ and $\mathfrak{g}_{1}^{\dagger}=1 /(x \mathfrak{e})$. But $\mathfrak{g}_{1} \preccurlyeq \mathfrak{e}$, so $\mathfrak{g} \preccurlyeq \mathfrak{g}_{1}$, and $\asymp$ would mean $\mathfrak{g}_{1}=\mathfrak{e}$ and $\mathfrak{g}_{1}^{\dagger}=1 /(x \mathfrak{e})$, so $\mathfrak{e}^{\dagger} \asymp 1 /(x \mathfrak{e})$, which is false; thus $\mathfrak{g} \prec \mathfrak{g}_{1}$. Also, $\mathfrak{g}_{2} \preccurlyeq \mathfrak{e}$, so $\mathfrak{g} \preccurlyeq \mathfrak{g}_{2}$. This time $\asymp$ means $\mathfrak{g}_{2}=\mathfrak{e}, \mathfrak{g}_{1}=\mathfrak{g}, \mathfrak{g}^{\dagger} \asymp 1 /(x \mathfrak{e})$. 
Thus we may use equation (16) to recursively define $\alpha_{\mathfrak{g}}(s)$. Indeed, solving the differential equation, we get

$$
\alpha_{\mathfrak{g}}(s)=\frac{e^{a b s}-1}{e^{a b}-1}\left(c_{\mathfrak{g}}-\int_{0}^{1} e^{a b(1-u)} f_{\mathfrak{g}}(u) d u\right)+\int_{0}^{s} e^{a b(s-u)} f_{\mathfrak{g}}(u) d u
$$

if $\mathfrak{g}^{\dagger} \sim b /(x \mathfrak{e})$.

Theorem 3.9. If (log-free) $T \sim x$ is deep, then it does not admit a real iteration group of the form (12).

Proof. The proof is as in Proposition 2.6 above. Here are the details. Write $T=$ $x(1+U), U \sim a \mathfrak{e}, \mathfrak{e} \prec 1$. Suppose

$$
\Phi(s, x)=x\left(1+\sum_{\mathfrak{g} \in \mathfrak{B}} \alpha_{\mathfrak{g}}(s) \mathfrak{g}\right)
$$

satisfies (41), (2), $\Phi(1, x)=T(x)$, and $\mathfrak{B}$ is a subgrid ( $\mathrm{W}$ well ordered). We may assume $\alpha_{\mathfrak{g}} \neq 0$ for all $\mathfrak{g} \in \mathfrak{B}$. Write

$$
\mathfrak{B}_{1}=\left\{\mathfrak{g} \in \mathfrak{B}: \mathfrak{g}^{\dagger} \preccurlyeq 1 /(x \mathfrak{e})\right\}, \quad \mathfrak{B}_{2}=\left\{\mathfrak{g} \in \mathfrak{B}: \mathfrak{g}^{\dagger} \succ 1 /(x \mathfrak{e})\right\}
$$

(the moderate and deep portions of $\mathfrak{B})$. As before, $\max \mathfrak{B}=\mathfrak{e}$ and $\alpha_{\mathfrak{e}}(s)=a s$, $\alpha_{\mathfrak{e}}^{\prime}(0)=a, a \neq 0$.

Let $\mathfrak{m}=\max \mathfrak{B}_{2}$. So $\mathfrak{m}^{\dagger} \succ 1 /(x \mathfrak{e})$. Write $\mathfrak{m}^{\dagger} \sim-b \mathfrak{p} /(x \mathfrak{e}), b \in \mathbb{R}, b>0$, $\mathfrak{p} \in \mathfrak{G}, \mathfrak{p} \succ 1$. ( $\mathfrak{m}$ is small and positive, so $\mathfrak{m}^{\prime}$ is negative.) In the AczélJabotinsky Equation (44) we will consider the coefficient of $x \mathfrak{p m}=x \operatorname{mag}\left((x \mathfrak{m})^{\prime} \mathfrak{e}\right)$. By Lemma 3.11 $(\mathrm{g}),\left((x \mathfrak{m})^{\prime}\right)^{\dagger} \sim \mathfrak{m}^{\dagger} \succ 1 /(x \mathfrak{e})$; by Remark 3.4, $\mathfrak{e}^{\dagger} \prec 1 /(x \mathfrak{e})$; so $(\mathfrak{p m})^{\dagger}=\left((x \mathfrak{m})^{\prime}\right)^{\dagger}+\mathfrak{e}^{\dagger} \sim\left((x \mathfrak{m})^{\prime}\right)^{\dagger} \succ 1 /(x \mathfrak{e})$ and $\mathfrak{p m} \notin \mathfrak{B}_{1}$. Also, $\mathfrak{p m} \succ \mathfrak{m}$, so $\mathfrak{p m} \notin \mathfrak{B}_{2}$. Thus $\mathfrak{p m} \notin \mathfrak{B}$, so $\alpha_{\mathfrak{p m}}=0$.

We claim that the only $\mathfrak{g}_{1}, \mathfrak{g}_{2} \in \mathfrak{B}$ with $\mathfrak{p m} \in \operatorname{supp}\left(\left(x \mathfrak{g}_{1}\right)^{\prime} \mathfrak{g}_{2}\right)$ are $\mathfrak{g}_{1}=\mathfrak{m}, \mathfrak{g}_{2}=\mathfrak{e}$. To prove this, consider these cases:

(a) $\mathfrak{g}_{1} \succ \mathfrak{m}, \mathfrak{g}_{2} \succ \mathfrak{m}$. By Lemma $3.14(\mathrm{~b}), \operatorname{supp}\left(\left(x \mathfrak{g}_{1}\right)^{\prime}\right) \subseteq \mathfrak{B}_{1}$, and by Lemma 3.14(a), $\operatorname{supp}\left(\left(x \mathfrak{g}_{1}\right)^{\prime} \mathfrak{g}_{2}\right) \subseteq \mathfrak{B}_{1}$. So $\mathfrak{p m} \notin \operatorname{supp}\left(\left(x \mathfrak{g}_{1}\right)^{\prime} \mathfrak{g}_{2}\right)$.

(b) $\mathfrak{g}_{1}=\mathfrak{m}, \mathfrak{g}_{2}=\mathfrak{e}$. In this case $\mathfrak{p m}=\operatorname{mag}\left(\left(x \mathfrak{g}_{1}\right)^{\prime} \mathfrak{g}_{2}\right)$, so $\mathfrak{p m} \in \operatorname{supp}\left(\left(x \mathfrak{g}_{1}\right)^{\prime} \mathfrak{g}_{2}\right)$.

(c) $\mathfrak{g}_{1}=\mathfrak{m}, \mathfrak{g}_{2} \prec \mathfrak{e}$. Then $\left(x \mathfrak{g}_{1}\right)^{\prime} \mathfrak{g}_{2} \prec(x \mathfrak{m})^{\prime} \mathfrak{e} \asymp \mathfrak{p m}$, so $\mathfrak{p m} \notin \operatorname{supp}\left(\left(x \mathfrak{g}_{1}\right)^{\prime} \mathfrak{g}_{2}\right)$.

(d) $\mathfrak{g}_{1} \prec \mathfrak{m}$. Then $\left(x \mathfrak{g}_{1}\right)^{\prime} \mathfrak{g}_{2} \prec(x \mathfrak{m})^{\prime} \mathfrak{e} \asymp \mathfrak{p m}$, so $\mathfrak{p m} \notin \operatorname{supp}\left(\left(x \mathfrak{g}_{1}\right)^{\prime} \mathfrak{g}_{2}\right)$.

(e) $\mathfrak{g}_{2} \preccurlyeq \mathfrak{m}$. Then by Remark 3.4 . $\left(x \mathfrak{g}_{1}\right)^{\prime} \mathfrak{g}_{2} \preccurlyeq(x \mathfrak{e})^{\prime} \mathfrak{m} \prec \mathfrak{m} \prec \mathfrak{p m}$, so $\mathfrak{p m} \notin$ $\operatorname{supp}\left(\left(x \mathfrak{g}_{1}\right)^{\prime} \mathfrak{g}_{2}\right)$.

So consideration of the coefficient of $x \mathrm{pm}$ in (41) yields

$$
0=-b \alpha_{\mathfrak{m}}(s) \alpha_{\mathfrak{e}}^{\prime}(0)=-a b \alpha_{\mathfrak{m}}(s),
$$

where $-a b \neq 0$, so $\alpha_{\mathfrak{m}}=0$, a contradiction.

Remark 3.10. If $T \sim x$, then a calculation shows that $T$ is shallow, moderate, or deep if and only if $\log \circ T \circ \exp$ is shallow, moderate, or deep, respectively. This invariance of the classification will show that the three theorems above are correct for all $T \in \mathbb{T}$ with $T \sim x$, even if not $\log$-free. For example, $T=x+\log x$ is shallow, since log-free

$$
\log \circ T \circ \exp =x+\sum_{j=1}^{\infty} \frac{(-1)^{j+1}}{j} x^{j} e^{-j x}
$$

is shallow. 
Recall that if $T$ is large and positive and has exponentiality 0 , then for some $k$ we have $\log ^{[k]} \circ T \circ \exp ^{[k]} \sim x$. We might try to use the same principle to extend the definitions of shallow, moderate, and deep to such transseries $T$ even if $T \not x$ : Define $T$ as shallow provided $\log ^{[k]} \circ T \circ \exp ^{[k]}$ is shallow for some $k$; similarly for moderate and deep. Examples: $T=x \log x$ is shallow, since $\log \circ T \circ \exp =x+\log x$ is shallow. The finite power series $U=2 x-2 / x$ is moderate, since

$$
\log \circ U \circ \exp =x+\log 2-\sum_{j=1}^{\infty} \frac{e^{-2 j x}}{j}
$$

is moderate. Also, $V=2 x-2 e^{-x}$ is deep.

But the usefulness of this extension is not entirely clear, since it may produce a family $\Phi(s, x)$ without common support. Example: For $T=x \log x$ we compute $S=\log \circ T \circ \exp =x+\log x$. So we get a real iteration group for $S$ of the form $\Psi(s, x)=x+s \log x+\mathrm{o}(1)$, and then a real iteration group for $T$ of the form $\Phi(s, x)=\exp (\Psi(s, \log x))=x(\log x)^{s}+\cdots$. These are not all supported by a common subgrid or even a common well ordered set. When the support depends on the parameter $s$, it may no longer make sense to require that the coefficients be differentiable.

Example $x^{2}+c$ is also deep. It is discussed in Section 6, below. The figure there illustrates supports of iterates $M^{[s]}$ that vary with $s$.

\section{Technical lemmas.}

Lemma 3.11. Let $\mathfrak{a}, \mathfrak{b} \in \mathfrak{G}$ and $A, B \in \mathbb{T}$. (a) If $\mathfrak{a}=e^{A}$, with $A \neq 0$ purely large, then $\mathfrak{a}^{\dagger}=A^{\prime}$. (b) $(A B)^{\dagger}=A^{\dagger}+B^{\dagger}$. (c) If $A=a \mathfrak{g}(1+U), \mathfrak{g} \neq 1, U \prec 1$, then $A^{\dagger} \sim \mathfrak{g}^{\dagger}$. (d) If $1 \prec \mathfrak{a} \preccurlyeq \mathfrak{b}$, then $\mathfrak{a}^{\dagger} \preccurlyeq \mathfrak{b}^{\dagger}$. ( $\left.\mathrm{d}^{\prime}\right)$ If $1 \prec A \preccurlyeq B$, then $A^{\dagger} \preccurlyeq B^{\dagger}$. (e) If $1 \succ \mathfrak{a} \succcurlyeq \mathfrak{b}$, then $\mathfrak{a}^{\dagger} \preccurlyeq \mathfrak{b}^{\dagger}$. (e $\left.\mathrm{e}^{\prime}\right)$ If $1 \succ A \succcurlyeq B$, then $A^{\dagger} \preccurlyeq B^{\dagger}$. (f) If $\mathfrak{b} \prec \mathfrak{a} \prec 1 / \mathfrak{b}$, then $\mathfrak{a}^{\dagger} \preccurlyeq \mathfrak{b}^{\dagger}$. (g) If $\mathfrak{b} \neq 1$ is log-free and $\mathfrak{n} \in \operatorname{supp}\left((x \mathfrak{b})^{\prime}\right)$, then $\mathfrak{n}^{\dagger} \sim \mathfrak{b}^{\dagger}$.

Proof. (a) $\mathfrak{a}^{\prime}=A^{\prime} e^{A}$, so $\mathfrak{a}^{\dagger}=\mathfrak{a}^{\prime} / \mathfrak{a}=A^{\prime}$.

(b) Product rule.

(c) Since $\mathfrak{g}=e^{L}, L$ purely large, note $\mathfrak{g}^{\dagger}=L^{\prime}$. Also, $U \prec 1$, so $(1+U)^{\dagger}=$ $U^{\prime} /(1+U) \sim U^{\prime}$. Now $L \succ 1 \succ U$, so $L^{\prime} \succ U^{\prime}$ and $\mathfrak{g}^{\dagger} \succ(1+U)^{\dagger}$. So $A^{\dagger}=$ $a^{\dagger}+\mathfrak{g}^{\dagger}+(1+U)^{\dagger} \sim \mathfrak{g}^{\dagger}$.

(d) Write $\mathfrak{a}=e^{A}, \mathfrak{b}=e^{B}$, with $A, B$ purely large. Now $0<A \leq B$, so $A \preccurlyeq B$. Also, $B \nsucc 1$, so $A^{\prime} \preccurlyeq B^{\prime}$, that is, $\mathfrak{a}^{\dagger} \preccurlyeq \mathfrak{b}^{\dagger}$. ( (d') follows from (c).

(e) Write $\mathfrak{a}=e^{A}, \mathfrak{b}=e^{B}$, with $A, B$ purely large. Now $0>A \geq B$, so $A \preccurlyeq B$. Also, $B \nprec 1$, so $A^{\prime} \preccurlyeq B^{\prime}$, that is, $\mathfrak{a}^{\dagger} \preccurlyeq \mathfrak{b}^{\dagger}$. ( (e') follows from (c).

(f) If $\mathfrak{a}=1$, then $\mathfrak{a}^{\dagger}=0$. If $\mathfrak{a} \prec 1$, then apply (e). If $\mathfrak{a} \succ 1$, note that $\mathfrak{b}^{\dagger}=-(1 / \mathfrak{b})^{\dagger} \asymp(1 / \mathfrak{b})^{\dagger}$, then apply $(\mathrm{d})$.

(g) Case $1: \mathfrak{b}=x^{b}, b \in \mathbb{R}, b \neq 0$. Then $x \mathfrak{b}=x^{b+1},(x \mathfrak{b})^{\prime}=(b+1) x^{b} \sim \mathfrak{b}$. So $\left((x \mathfrak{b})^{\prime}\right)^{\dagger} \sim \mathfrak{b}^{\dagger}$. Case 2: $\mathfrak{b}=x^{b} e^{L}, L \neq 0$ has height $n \geq 0$. Assume $\mathfrak{b} \prec 1$; the case $\mathfrak{b} \succ 1$ is similar. Then $x \mathfrak{b}=x^{b+1} e^{L},(x \mathfrak{b})^{\prime}=\left((b+1)+x L^{\prime}\right) x^{b} e^{L}=A \mathfrak{b}$, where $A=(b+1)+x L^{\prime}$ has height $n$. Now $\mathfrak{n} \in \operatorname{supp}\left((x \mathfrak{b})^{\prime}\right)$, so $\mathfrak{n}=\mathfrak{a} \mathfrak{b}$ where $\mathfrak{a} \in \operatorname{supp} A$. But $\mathfrak{a}$ has height $n$, so by "height wins" [11, Prop. 3.72], we have $\mathfrak{b} \prec \mathfrak{a} \prec 1 / \mathfrak{b}$. In the proofs of (d) and (e), if it is a case of "height wins", then we get strict inequality $A \prec B$ and $\mathfrak{a}^{\dagger} \prec \mathfrak{b}^{\dagger}$. So here $\mathfrak{a}^{\dagger} \prec \mathfrak{b}^{\dagger}$. Therefore $\mathfrak{n}^{\dagger}=(\mathfrak{a} \mathfrak{b})^{\dagger}=\mathfrak{a}^{\dagger}+\mathfrak{b}^{\dagger} \sim \mathfrak{b}^{\dagger}$. 
Lemma 3.12. Let $T=x(1+a \mathfrak{e}+\mathrm{o}(\mathfrak{e}))$ with $\mathfrak{e} \in \mathfrak{G}, \mathfrak{e} \prec 1, a \in \mathbb{R}, a>0$. Let $\mathfrak{g} \in \mathfrak{G}$, $\mathfrak{g} \prec 1$. Then (i) $\mathfrak{e}(T) \sim \mathfrak{e}$; (ii) if $\mathfrak{g}^{\dagger} \prec 1 /(x \mathfrak{e})$, then $\mathfrak{g}(T) \sim \mathfrak{g}$; (iii) if $\mathfrak{g}^{\dagger} \sim-b /(x \mathfrak{e}$ ), $b \in \mathbb{R}$, then $\mathfrak{g}(T) \sim e^{-a b} \mathfrak{g}$; (iv) if $\mathfrak{g}^{\dagger} \succ 1 /(x \mathfrak{e})$, then $\mathfrak{g}(T) \prec \mathfrak{g}$.

Proof. (i) As in Remark 3.4 $\mathfrak{e}^{\prime} \prec 1 / x$. Then

$$
\mathfrak{e}(T)-\mathfrak{e}=\int_{x}^{T} \mathfrak{e}^{\prime} \prec \int_{x}^{T} \frac{1}{x}=\log \frac{T}{x}=\log (1+a \mathfrak{e}+\mathrm{o}(\mathfrak{e})) \sim a \mathfrak{e} \asymp \mathfrak{e},
$$

SO $\mathfrak{e}(T) \sim \mathfrak{e}$.

(ii) Assume $\mathfrak{g}^{\dagger} \prec 1 /(x \mathfrak{e})$. Then

$$
\log \frac{\mathfrak{g}(T)}{\mathfrak{g}}=\int_{x}^{T} \mathfrak{g}^{\dagger} \prec \int_{x}^{T} \frac{1}{x \mathfrak{e}} .
$$

By Proposition 0.5, the value of this integral is between

$$
\frac{T-x}{x \mathfrak{e}} \sim \frac{a x \mathfrak{e}}{x \mathfrak{e}}=a \quad \text { and } \quad \frac{T-x}{T \mathfrak{e}(T)} \sim \frac{a x \mathfrak{e}}{x \mathfrak{e}}=a .
$$

Thus $\log (\mathfrak{g}(T) / \mathfrak{g}) \prec 1$, so $\mathfrak{g}(T) / \mathfrak{g} \sim 1$.

(iii) Assume $\mathfrak{g}^{\dagger} \sim-b /(x \mathfrak{e})$. Then

$$
\log \frac{\mathfrak{g}(T)}{\mathfrak{g}}=\int_{x}^{T} \mathfrak{g}^{\dagger} \sim \int_{x}^{T} \frac{-b}{x \mathfrak{e}} \sim-a b,
$$

where the integral was estimated in the same way as in (ii). Therefore $\mathfrak{g}(T) \sim e^{-a b} \mathfrak{g}$.

(iv) Assume $\mathfrak{g}^{\dagger} \succ 1 /(x \mathfrak{e})$. Then

$$
\log \frac{\mathfrak{g}(T)}{\mathfrak{g}(x)}=\int_{x}^{T} \mathfrak{g}^{\dagger} \succ \int_{x}^{T} \frac{1}{x \mathfrak{e}} \sim a .
$$

But $\log (\mathfrak{g}(T) / \mathfrak{g}(x))<0$, so $\mathfrak{g}(T) / \mathfrak{g}(x) \prec 1$ and $\mathfrak{g}(T) \prec \mathfrak{g}$.

[Note this proof uses Proposition 0.5, which is not proved in [12.]

For completeness, we note the following analogous version for $T<x$.

Lemma 3.13. Let $T=x(1-a \mathfrak{e}+\mathrm{o}(\mathfrak{e}))$ with $\mathfrak{e} \in \mathfrak{G}, \mathfrak{e} \prec 1, a \in \mathbb{R}, a>0$. Let $\mathfrak{g} \in \mathfrak{G}$, $\mathfrak{g} \prec 1$. Then (i) $\mathfrak{e}(T) \sim \mathfrak{e}$; (ii) if $\mathfrak{g}^{\dagger} \prec 1 /(x \mathfrak{e})$, then $\mathfrak{g}(T) \sim \mathfrak{g}$; (iii) if $\mathfrak{g}^{\dagger} \sim-b /(x \mathfrak{e})$, $b \in \mathbb{R}$, then $\mathfrak{g}(T) \sim e^{a b} \mathfrak{g} ;$ (iv) if $\mathfrak{g}^{\dagger} \succ 1 /(x \mathfrak{e})$, then $\mathfrak{g}(T) \succ \mathfrak{g}$.

Lemma 3.14. Let $\mathfrak{m} \in \mathfrak{G}$ be a monomial. Let $\mathfrak{B}=\left\{\mathfrak{g} \in \mathfrak{G}: \mathfrak{g}^{\dagger} \prec \mathfrak{m}\right\}$ and $\widetilde{\mathfrak{B}}=$ $\left\{\mathfrak{g} \in \mathfrak{G}: \mathfrak{g}^{\dagger} \preccurlyeq \mathfrak{m}\right\}$. Then: (a) $\mathfrak{B}$ and $\widetilde{\mathfrak{B}}$ are subgroups of $\mathfrak{G}$. (b) Let $\mathfrak{g} \in \mathfrak{G}$ be logfree and small. If $\mathfrak{g} \in \mathfrak{B}$, then $\operatorname{supp}\left((x \mathfrak{g})^{\prime}\right) \subseteq \mathfrak{B}$. If $\mathfrak{g} \in \widetilde{\mathfrak{B}}$, then $\operatorname{supp}\left((x \mathfrak{g})^{\prime}\right) \subseteq \widetilde{\mathfrak{B}}$. (c) Let $\mathfrak{g}=x^{b} e^{L}$, where $L$ is purely large and log-free. If $\mathfrak{g} \in \mathfrak{B}$, then $\operatorname{supp} L \subseteq \mathfrak{B}$. If $\mathfrak{g} \in \widetilde{\mathfrak{B}}$, then $\operatorname{supp} L \subseteq \widetilde{\mathfrak{B}}$.

Proof. (a) $(1 / \mathfrak{g})^{\dagger}=-\mathfrak{g}^{\dagger}$, so if $\mathfrak{g} \in \mathfrak{B}$, then $1 / \mathfrak{g} \in \mathfrak{B}$, and if $\mathfrak{g} \in \widetilde{\mathfrak{B}}$, then $1 / \mathfrak{g} \in \widetilde{\mathfrak{B}}$. Also, $\left(\mathfrak{g}_{1} \mathfrak{g}_{2}\right)^{\dagger}=\mathfrak{g}_{1}^{\dagger}+\mathfrak{g}_{2}^{\dagger}$, so if $\mathfrak{g}_{1}, \mathfrak{g}_{2} \in \mathfrak{B}$, then $\mathfrak{g}_{1} \mathfrak{g}_{2} \in \mathfrak{B}$, and if $\mathfrak{g}_{1}, \mathfrak{g}_{2} \in \widetilde{\mathfrak{B}}$, then $\mathfrak{g}_{1} \mathfrak{g}_{2} \in \widetilde{\mathfrak{B}}$.

(b) Apply Lemma 3.11(g).

(c) Let $\mathfrak{n} \in \operatorname{supp} L$. Then by "height wins" we have $\mathfrak{n}^{\dagger} \preccurlyeq \mathfrak{g}^{\dagger}$. 
Lemma 3.15. $\mathrm{G}$ Let $\mathfrak{A}$ be a subgrid. Then $\bigcup_{\mathfrak{a} \in \mathfrak{A}} \operatorname{supp}\left(\mathfrak{a}^{\prime}\right)$ is also a subgrid. For any given $\mathfrak{g} \in \mathfrak{G}$, there are only finitely many $\mathfrak{a} \in \mathfrak{A}$ with $\mathfrak{g} \in \operatorname{supp}\left(\mathfrak{a}^{\prime}\right)$.

Proof. [17, Prop. 5.2], 9, Props. 7.3, 7.12, 7.13], 13, Rem. 4.6]. (This is exactly what is needed for the proof that the derivative $\left(\sum_{\mathfrak{a} \in \mathfrak{A}} \mathfrak{a}\right)^{\prime}$ is defined.)

Lemma 3.16. W Let $\mathfrak{A}$ be well ordered. Then $\bigcup_{\mathfrak{a} \in \mathfrak{A}} \operatorname{supp}\left(\mathfrak{a}^{\prime}\right)$ is also well ordered. For any given $\mathfrak{g} \in \mathfrak{G}$, there are only finitely many $\mathfrak{a} \in \mathfrak{A}$ with $\mathfrak{g} \in \operatorname{supp}\left(\mathfrak{a}^{\prime}\right)$.

Proof. [9, Lem. 3.2] or [12, Prop. 2.5].

Lemma 3.17. $\mathrm{G}$ Let $\mathfrak{A}$ and $\mathfrak{B}$ be subgrids. Then $\mathfrak{A} \mathfrak{B}$ is a subgrid. If $\mathfrak{g} \in \mathfrak{A} \mathfrak{B}$, then there are only finitely many pairs $\mathfrak{a} \in \mathfrak{A}, \mathfrak{b} \in \mathfrak{B}$ with $\mathfrak{a} \mathfrak{b}=\mathfrak{g}$.

Proof. [11, Prop. 3.35(d)] and [11, Prop. 3.27].

Lemma 3.18. $\mathrm{W}$ Let $\mathfrak{A}$ and $\mathfrak{B}$ be well ordered. Then $\mathfrak{A} \mathfrak{B}$ is well ordered. If $\mathfrak{g} \in \mathfrak{A} \mathfrak{B}$, then there are only finitely many pairs $\mathfrak{a} \in \mathfrak{A}, \mathfrak{b} \in \mathfrak{B}$ with $\mathfrak{a} \mathfrak{b}=\mathfrak{g}$.

Proof. [11, Prop. 3.27].

Lemma 3.19. $G$ Let $\mathfrak{B}$ be a subgrid. Let $\mathfrak{g} \in \mathfrak{G}$. There are only finitely many pairs $\mathfrak{g}_{1}, \mathfrak{g}_{2} \in \mathfrak{B}$ such that $\mathfrak{g} \in \operatorname{supp}\left(\left(x \mathfrak{g}_{1}\right)^{\prime} \mathfrak{g}_{2}\right)$.

Proof. Since $\mathfrak{B}$ is a subgrid, $\mathfrak{B}_{1}:=\{x \mathfrak{g}: \mathfrak{g} \in \mathfrak{B}\}$ is also a subgrid. By Lemma 3.15, $\mathfrak{B}_{2}:=\bigcup_{\mathfrak{g} \in \mathfrak{B}} \operatorname{supp}\left((x \mathfrak{g})^{\prime}\right)$ is a subgrid. Then $\mathfrak{B}_{2} \mathfrak{B}=\bigcup_{\mathfrak{g}_{1}, \mathfrak{g}_{2} \in \mathfrak{B}} \operatorname{supp}\left(\left(x \mathfrak{g}_{1}\right)^{\prime} \mathfrak{g}_{2}\right)$, and by Lemma 3.17, for any given $\mathfrak{g} \in \mathfrak{G}$, there are only finitely many pairs $\mathfrak{g}_{1}, \mathfrak{g}_{2} \in \mathfrak{B}$ such that $\mathfrak{g} \in \operatorname{supp}\left(\left(x \mathfrak{g}_{1}\right)^{\prime} \mathfrak{g}_{2}\right)$.

Lemma 3.20. W Let $\mathfrak{B}$ be well ordered. Let $\mathfrak{g} \in \mathfrak{G}$. There are only finitely many pairs $\mathfrak{g}_{1}, \mathfrak{g}_{2} \in \mathfrak{B}$ such that $\mathfrak{g} \in \operatorname{supp}\left(\left(x \mathfrak{g}_{1}\right)^{\prime} \mathfrak{g}_{2}\right)$.

Proof. Since $\mathfrak{B}$ is well ordered, $\mathfrak{B}_{1}:=\{x \mathfrak{g}: \mathfrak{g} \in \mathfrak{B}\}$ is also well ordered. By Lemma 3.16, $\mathfrak{B}_{2}:=\bigcup_{\mathfrak{g} \in \mathfrak{B}} \operatorname{supp}\left((x \mathfrak{g})^{\prime}\right)$ is well ordered, and each monomial in $\mathfrak{B}_{2}$ belongs to $\operatorname{supp}\left((x \mathfrak{g})^{\prime}\right)$ for only finitely many $\mathfrak{g}$. Then $\mathfrak{B}_{2} \mathfrak{B}=\bigcup_{\mathfrak{g}_{1}, \mathfrak{g}_{2} \in \mathfrak{B}} \operatorname{supp}\left(\left(x \mathfrak{g}_{1}\right)^{\prime} \mathfrak{g}_{2}\right)$, and by Lemma 3.18, for any given $\mathfrak{g} \in \mathfrak{G}$, there are only finitely many pairs $\mathfrak{g}_{2}, \mathfrak{g}_{2} \in \mathfrak{B}$ such that $\mathfrak{g} \in \operatorname{supp}\left((x \mathfrak{g}) ; \mathfrak{g}_{2}\right)$.

Lemma 3.21. Let $\mathfrak{e} \in \mathfrak{G}, \mathfrak{e} \prec 1$ and let

$$
\mathfrak{A}=\left\{\mathfrak{g} \in \mathfrak{G}: \mathfrak{g} \preccurlyeq \mathfrak{e}, \mathfrak{g}^{\dagger} \prec \frac{1}{x \mathfrak{e}}\right\}, \quad \widetilde{\mathfrak{A}}=\left\{\mathfrak{g} \in \mathfrak{G}: \mathfrak{g} \preccurlyeq \mathfrak{e}, \mathfrak{g}^{\dagger} \preccurlyeq \frac{1}{x \mathfrak{e}}\right\} .
$$

(a) If $\mathfrak{g}_{1}, \mathfrak{g}_{2} \in \mathfrak{A}$, then $\mathfrak{g}_{1} \mathfrak{g}_{2} \in \mathfrak{A}$. If $\mathfrak{g}_{1}, \mathfrak{g}_{2} \in \widetilde{\mathfrak{A}}$, then $\mathfrak{g}_{1} \mathfrak{g}_{2} \in \widetilde{\mathfrak{A}}$. (b) If $\mathfrak{g}_{1}, \mathfrak{g}_{2} \in \mathfrak{A}$, then $\operatorname{supp}\left(\left(x \mathfrak{g}_{1}\right)^{\prime} \mathfrak{g}_{2}\right) \subseteq \mathfrak{A}$. If $\mathfrak{g}_{1}, \mathfrak{g}_{2} \in \widetilde{\mathfrak{A}}$, then $\operatorname{supp}\left(\left(x \mathfrak{g}_{1}\right)^{\prime} \mathfrak{g}_{2}\right) \subseteq \widetilde{\mathfrak{A}}$. (c) If $\mathfrak{g} \in \mathfrak{A}$, then $\operatorname{supp}\left(x \mathfrak{e} \mathfrak{g}^{\prime}\right) \subseteq \mathfrak{A}$. If $\mathfrak{g} \in \widetilde{\mathfrak{A}}$, then $\operatorname{supp}\left(x \mathfrak{e} \mathfrak{g}^{\prime}\right) \subseteq \widetilde{\mathfrak{A}}$.

Proof. (a) If $\mathfrak{g}_{1}, \mathfrak{g}_{2} \preccurlyeq \mathfrak{e}$, then $\mathfrak{g}_{1} \mathfrak{g}_{2} \preccurlyeq \mathfrak{e} \mathfrak{e} \prec \mathfrak{e}$. Combine this with Lemma 3.14(a).

(b) If $\mathfrak{g}_{1} \preccurlyeq \mathfrak{e}$, then $x \mathfrak{g}_{1} \preccurlyeq x \mathfrak{e}$ and $\left(x \mathfrak{g}_{1}\right)^{\prime} \preccurlyeq(x \mathfrak{e})^{\prime}$. Now $\mathfrak{e} \prec 1$, so $x \mathfrak{e} \prec x$ and $(x \mathfrak{e})^{\prime} \prec 1$. If $\mathfrak{g}_{2} \preccurlyeq \mathfrak{e}$ also, then $\left(x \mathfrak{g}_{1}\right)^{\prime} \mathfrak{g}_{2} \preccurlyeq(x \mathfrak{e})^{\prime} \mathfrak{e} \prec 1 \mathfrak{e}=\mathfrak{e}$. Combine this with Lemma 3.14(b).

(c) is similar, noting that $\mathfrak{e}^{\dagger} \prec 1 /(x \mathfrak{e})$ and $x^{\dagger} \prec 1 /(x \mathfrak{e})$ 
Lemma 3.22. $\mathrm{G}$ Let $\mathfrak{B} \subset \mathfrak{G}^{\text {small }}$ be a log-free subgrid. Write $\mathfrak{e}=\max \mathfrak{B}$ and assume $\mathfrak{B} \subseteq\left\{\mathfrak{g} \in \mathfrak{G}: \mathfrak{g} \preccurlyeq \mathfrak{e}, \mathfrak{g}^{\dagger} \preccurlyeq 1 /(x \mathfrak{e})\right\}$. Let $\widetilde{\mathfrak{B}}$ be the least subset of $\mathfrak{G}$ such that

(i) $\widetilde{\mathfrak{B}} \supseteq \mathfrak{B}$,

(ii) if $\mathfrak{g}_{1}, \mathfrak{g}_{2} \in \widetilde{\mathfrak{B}}$, then $\mathfrak{g}_{1} \mathfrak{g}_{2} \in \widetilde{\mathfrak{B}}$,

(iii) if $\mathfrak{g}_{1}, \mathfrak{g}_{2} \in \widetilde{\mathfrak{B}}$, then $\operatorname{supp}\left(\left(x \mathfrak{g}_{1}\right)^{\prime} \mathfrak{g}_{2}\right) \subseteq \widetilde{\mathfrak{B}}$.

Then $\widetilde{\mathfrak{B}}$ is a subgrid.

Proof. Let $\mathfrak{A}$ be the least subset of $\mathfrak{G}$ such that

(i) $\mathfrak{A} \supseteq \mathfrak{B}$,

(ii) if $x^{b} e^{L} \in \mathfrak{A}$, then $\operatorname{supp} L \subseteq \mathfrak{A}$.

By [11, Prop. 2.21], $\mathfrak{A}$ is a subgrid. From Lemma 3.14(c) we have $\mathfrak{g}^{\dagger} \preccurlyeq 1 /(x \mathfrak{e})$ for all $\mathfrak{g} \in \mathfrak{A}$. There is a ratio set $\boldsymbol{\mu}=\left\{\mu_{1}, \cdots, \mu_{n}\right\}$, chosen from the group generated by $\mathfrak{A}$, so that $\mathfrak{A} \subseteq \mathfrak{J}^{\mu}$. Because they come from the group generated by $\mathfrak{A}$, we have $\mu_{i}^{\dagger} \preccurlyeq 1 /(x \mathfrak{e})$ for $1 \leq i \leq n$ by Lemma 3.14(a). Remark that $x^{\dagger} \prec 1 /(x \mathfrak{e})$ since $\mathfrak{e} \prec 1$. Also, $\mathfrak{e}^{\dagger} \prec 1 /(x \mathfrak{e})$ was noted in Remark 3.4. So we may without harm add more generators to $\boldsymbol{\mu}$ and assume $\mathfrak{e}, x \in \mathfrak{J}^{\mu}$. This has been arranged so that if $\mathfrak{g} \in \mathfrak{J}^{\mu}$, then $\operatorname{supp}\left(\mathfrak{g}^{\prime}\right) \subseteq \mathfrak{J}^{\mu}$. Now $x \mathfrak{e} \mu_{i}^{\dagger} \preccurlyeq 1$, so

$$
\left\{\mathfrak{e}, x^{-1}\right\} \cup \bigcup_{i=1}^{n} \operatorname{supp}\left(x \mathfrak{e} \mu_{i}^{\dagger}\right) \cup \frac{1}{\mathfrak{e}} \mathfrak{B}
$$

is a finite union of subgrids, so it is itself a subgrid. All of its elements are $\preccurlyeq 1$, so [11, Prop. 3.52] there is a ratio set $\boldsymbol{\alpha}$ such that $\mathfrak{J}^{\boldsymbol{\alpha}, \mathbf{0}}$ contains that finite union. Again all elements of $\boldsymbol{\alpha}$ may be chosen from the group generated by $\mathfrak{J}^{\mu}$. So all $\mathfrak{a} \in \mathfrak{J}^{\boldsymbol{\alpha}, \mathbf{0}}$ still satisfy $\mathfrak{a}^{\dagger} \preccurlyeq 1 /(x \mathfrak{e})$.

To complete the proof that $\widetilde{\mathfrak{B}}$ is a subgrid, we will show that $\widetilde{\mathfrak{B}} \subseteq \mathfrak{e} \mathfrak{J}^{\boldsymbol{\alpha}, \mathbf{0}}$. First, note that $\mathfrak{B} \subseteq \mathfrak{e} \mathfrak{J}^{\boldsymbol{\alpha}, \mathbf{0}}$. Next, if $\mathfrak{g}_{1}, \mathfrak{g}_{2} \in \mathfrak{e} \mathfrak{J}^{\boldsymbol{\alpha}, \mathbf{0}}$, then $\mathfrak{g}_{1} \mathfrak{g}_{2} \in \mathfrak{e}\left(\mathfrak{J}^{\boldsymbol{\alpha}, \mathbf{0}} \mathfrak{e} \mathfrak{J}^{\boldsymbol{\alpha}, \mathbf{0}}\right) \subseteq \mathfrak{e} \mathfrak{J}^{\boldsymbol{\alpha}, \mathbf{0}}$. Finally, suppose $\mathfrak{g}_{1}, \mathfrak{g}_{2} \in \mathfrak{e} \mathfrak{J}^{\boldsymbol{\alpha}, \boldsymbol{0}}$. Because $\boldsymbol{\alpha}$ is from the group $\mathfrak{J}^{\mu}$, we may write $\mathfrak{g}_{1}=\mu_{1}^{k_{1}} \cdots \mu_{n}^{k_{n}}$, and

$$
\begin{aligned}
\mathfrak{g}_{1}^{\dagger} & =k_{1} \mu_{1}^{\dagger}+\cdots+k_{n} \mu_{n}^{\dagger}, \\
x \mathfrak{e} \mathfrak{g}_{1}^{\dagger} & =k_{1} x \mathfrak{e} \mu_{1}^{\dagger}+\cdots+k_{n} x \mathfrak{e} \mu_{n}^{\dagger},
\end{aligned}
$$

so that $\operatorname{supp}\left(x \mathfrak{e} \mathfrak{g}_{1}^{\dagger}\right) \subseteq \mathfrak{J}^{\boldsymbol{\alpha}, \mathbf{0}}$. Also, $\mathfrak{g}_{1} / \mathfrak{e} \in \mathfrak{J}^{\boldsymbol{\alpha}, \mathbf{0}}$ and $\mathfrak{g}_{2} / \mathfrak{e} \in \mathfrak{J}^{\boldsymbol{\alpha}, \mathbf{0}}$. Therefore

$$
\operatorname{supp}\left(x \mathfrak{g}_{1}^{\prime} \mathfrak{g}_{2}\right)=\mathfrak{e}\left(\frac{\mathfrak{g}_{1}}{\mathfrak{e}}\right)\left(\frac{\mathfrak{g}_{2}}{\mathfrak{e}}\right) \operatorname{supp}\left(x \mathfrak{e} \mathfrak{g}_{1}^{\dagger}\right) \subseteq \mathfrak{e} \mathfrak{J}^{\boldsymbol{\alpha}, \mathbf{0}} .
$$

Also, $\left(x \mathfrak{g}_{1}\right)^{\prime} \mathfrak{g}_{2}=\mathfrak{g}_{1} \mathfrak{g}_{2}+x \mathfrak{g}_{1}^{\prime} \mathfrak{g}_{2}$, so $\operatorname{supp}\left(\left(x \mathfrak{g}_{1}\right)^{\prime} \mathfrak{g}_{2}\right) \subseteq \mathfrak{e} \mathfrak{J}^{\boldsymbol{\alpha}, \mathbf{0}}$. By the definition of $\widetilde{\mathfrak{B}}$ we have $\widetilde{\mathfrak{B}} \subseteq \mathfrak{e} \mathfrak{J}^{\boldsymbol{\alpha}, \mathbf{0}}$, and it is therefore a subgrid.

Lemma 3.23. $\mathrm{W}$ Let $\mathfrak{B} \subset \mathfrak{G}^{\text {small }}$ be log-free and well ordered. Write $\mathfrak{e}=\max \mathfrak{B}$ and assume $\mathfrak{B} \subseteq\left\{\mathfrak{g} \in \mathfrak{G}: \mathfrak{g} \preccurlyeq \mathfrak{e}, \mathfrak{g}^{\dagger} \preccurlyeq 1 /(x \mathfrak{e})\right\}$. Let $\widetilde{\mathfrak{B}}$ be the least subset of $\mathfrak{G}$ such that

(i) $\widetilde{\mathfrak{B}} \supseteq \mathfrak{B}$,

(ii) if $\mathfrak{g}_{1}, \mathfrak{g}_{2} \in \widetilde{\mathfrak{B}}$, then $\mathfrak{g}_{1} \mathfrak{g}_{2} \in \widetilde{\mathfrak{B}}$,

(iii) if $\mathfrak{g}_{1}, \mathfrak{g}_{2} \in \widetilde{\mathfrak{B}}$, then $\operatorname{supp}\left(\left(x \mathfrak{g}_{1}\right)^{\prime} \mathfrak{g}_{2}\right) \subseteq \widetilde{\mathfrak{B}}$.

Then $\widetilde{\mathfrak{B}}$ is well ordered.

Proof. Let $\mathfrak{B}_{1}$ be the least set such that $\mathfrak{B}_{1} \supseteq \mathfrak{B} \cup\left\{\mathfrak{e}^{2}\right\}$, and if $\mathfrak{g} \in \mathfrak{B}_{1}$, then $\operatorname{supp}\left(x \mathfrak{e g}^{\prime}\right) \subseteq \mathfrak{B}_{1}$. Then $\mathfrak{B}_{1}$ is well ordered by [12, Prop. 2.10]. For all $\mathfrak{g} \in \mathfrak{B}_{1}$ we 
have $\mathfrak{g} \preccurlyeq \mathfrak{e}$ and $\mathfrak{g}^{\dagger} \preccurlyeq 1 /(x \mathfrak{e})$ by Lemma $3.21(\mathrm{c})$. Still $\mathfrak{e}=\max \mathfrak{B}_{1}, \mathfrak{e}^{2} \in \mathfrak{B}_{1}$, and $\operatorname{supp}\left(x \mathfrak{e e}^{\prime}\right) \subseteq \mathfrak{B}_{1}$.

Let $\mathfrak{B}_{2}=\mathfrak{e}^{-1} \mathfrak{B}_{1}$. Then $\mathfrak{B}_{2}$ is well ordered, $\mathfrak{B}_{2} \supseteq \mathfrak{e}^{-1} \mathfrak{B}, 1=\max \mathfrak{B}_{2}, \mathfrak{e} \in \mathfrak{B}_{2}$, and $\operatorname{supp}\left(x \mathfrak{e}^{\prime}\right) \subseteq \mathfrak{B}_{2}$. If $\mathfrak{m} \in \mathfrak{B}_{2}$, then $\operatorname{supp}\left(x(\mathfrak{e m})^{\prime}\right) \subseteq \mathfrak{B}_{2}$.

Let $\mathfrak{B}_{3}$ be the semigroup generated by $\mathfrak{B}_{2}$. Then $\mathfrak{B}_{3}$ is well ordered, $\mathfrak{B}_{3} \supseteq \mathfrak{e}^{-1} \mathfrak{B}$, $1=\max \mathfrak{B}_{3}, \mathfrak{e} \in \mathfrak{B}_{3}$, and $\operatorname{supp}\left(x \mathfrak{e}^{\prime}\right) \subseteq \mathfrak{B}_{3}$. From the identity

$$
x\left(\mathfrak{e m}_{1} \mathfrak{m}_{2}\right)^{\prime}=x\left(\mathfrak{e m}_{1}\right)^{\prime} \cdot \mathfrak{m}_{2}+\mathfrak{m}_{1} \cdot x\left(\mathfrak{e m}_{2}\right)^{\prime}-x \mathfrak{e}^{\prime} \mathfrak{m}_{1} \mathfrak{m}_{2}
$$

we conclude: if $\mathfrak{m} \in \mathfrak{B}_{3}$, then $\operatorname{supp}\left(x(\mathfrak{e m})^{\prime}\right) \subseteq \mathfrak{B}_{3}$.

Finally, let $\mathfrak{B}_{4}=\mathfrak{e} \mathfrak{B}_{3}$. Then $\mathfrak{B}_{4}$ is well ordered, $\mathfrak{B}_{4} \supseteq \mathfrak{B}$, and $\mathfrak{e}=\max \mathfrak{B}_{4}$. Let $\mathfrak{g}_{1}, \mathfrak{g}_{2} \in \mathfrak{B}_{4}$. Then $\mathfrak{g}_{1} / \mathfrak{e}, \mathfrak{g}_{2} / \mathfrak{e} \in \mathfrak{B}_{3}$, so $\left(\mathfrak{g}_{1} / \mathfrak{e}\right) \cdot\left(\mathfrak{g}_{2} / \mathfrak{e}\right) \in \mathfrak{B}_{3}$ and $\mathfrak{g}_{1} \mathfrak{g}_{2} / \mathfrak{e}^{2} \in \mathfrak{B}_{3}$. Now $\mathfrak{e} \in \mathfrak{B}_{3}$, so $\mathfrak{g}_{1} \mathfrak{g}_{2} / \mathfrak{e} \in \mathfrak{B}_{3}$ and therefore $\mathfrak{g}_{1} \mathfrak{g}_{2} \in \mathfrak{B}_{4}$. Again let $\mathfrak{g}_{1}, \mathfrak{g}_{2} \in \mathfrak{B}_{4}$. Then $\mathfrak{g}_{1} / \mathfrak{e}, \mathfrak{g}_{2} / \mathfrak{e} \in \mathfrak{B}_{3}$. So $\operatorname{supp}\left(x \mathfrak{g}_{1}^{\prime}\right) \subseteq \mathfrak{B}_{3}$. Thus $\operatorname{supp}\left(x \mathfrak{g}_{1}^{\prime}\right) \mathfrak{g}_{2} / \mathfrak{e} \subseteq \mathfrak{B}_{3}, \operatorname{so} \operatorname{supp}\left(x \mathfrak{g}_{1}^{\prime}\right) \mathfrak{g}_{2} \subseteq$ $\mathfrak{B}_{4}$. Also, $\left(x \mathfrak{g}_{1}\right)^{\prime} \mathfrak{g}_{2}=\mathfrak{g}_{1} \mathfrak{g}_{2}+x \mathfrak{g}_{1}^{\prime} \mathfrak{g}_{2}$, so we conclude $\operatorname{supp}\left(\left(x \mathfrak{g}_{1}\right)^{\prime} \mathfrak{g}_{2}\right) \subseteq \mathfrak{B}_{4}$.

This shows $\mathfrak{B}_{4} \supseteq \widetilde{\mathfrak{B}}$, and therefore that $\widetilde{\mathfrak{B}}$ is well ordered.

\section{Abel's Equation}

Let $T \in \mathcal{P}, T>x$. Abel's Equation for $T$ is $V(T(x))=V(x)+1$. If a large positive $V$ exists satisfying this, then a real iteration group $\Phi$ may be obtained as $\Phi(s, x)=V^{[-1]} \circ(x+s) \circ V$. (In general such $\Phi$ will not have common support.) If $T \in \mathcal{P}, T<x$, then Abel's Equation for $T$ is $V(T(x))=V(x)-1$, and then we may similarly write $\Phi(s, x)=V^{[-1]} \circ(x-s) \circ V$.

We now do this in reverse: Let $\Phi(s, x)$ be of the form constructed as in Theorem 3.8, that is, coefficients defined recursively by (15) and (17). Then we can use $\Phi$ to get $V$ for Abel's Equation.

Theorem 4.1 (Moderate Abel). Let $T \sim x, T>x$ be moderate, and let $\Phi(s, x) \in \mathcal{P}$ for all $s \in \mathbb{R}$ be the real iteration group for $T$ constructed in Theorem 3.8 . Then

$$
V(x):=\int \frac{d x}{\Phi_{1}(0, x)}
$$

is large and positive and satisfies Abel's Equation $V(T(x))=V(x)+1$.

Proof. Now $V^{\prime} \sim 1 /(\operatorname{axe}) \succ 1 / x$, so $V \succ \log x$ is large. Also, $V^{\prime}>0$, so $V>0$. (A large negative transseries has negative derivative.)

From $\Phi(s+t, x)=\Phi(s, \Phi(t, x))$ take $\partial / \partial s$. Then substitute $t=1, s=0$ to get $\Phi_{1}(1, x)=\Phi_{1}(0, T)$. But $\Phi_{1}(s, x)=\Phi_{2}(s, x) \Phi_{1}(0, x)$. So $\Phi_{2}(1, x) \Phi_{1}(0, x)=$ $\Phi_{1}(1, x)=\Phi_{1}(0, T)$. Now from $\Phi(1, x)=T$ we have $\Phi_{2}(1, x)=T^{\prime}$. So

$$
\frac{T^{\prime}}{\Phi_{1}(0, T)}=\frac{1}{\Phi_{1}(0, x)}
$$

or $V^{\prime}(T) \cdot T^{\prime}=V^{\prime}(x)$, so $V(T)=V+c$ for some $c \in \mathbb{R}$. Now

$$
V(T)-V(x)=\int_{x}^{T} V^{\prime}=\int_{x}^{T} \frac{1}{\Phi_{1}(0, x)} \sim \int_{x}^{T} \frac{1}{a x \mathfrak{e}} .
$$

By Proposition 0.5, this integral is between

$$
\frac{T-x}{a x \mathfrak{e}} \sim \frac{a x \mathfrak{e}}{a x \mathfrak{e}}=1 \quad \text { and } \quad \frac{T-x}{a T \mathfrak{e}(T)} \sim \frac{a x \mathfrak{e}}{a x \mathfrak{e}}=1 .
$$

We used $\mathfrak{e}(T) \sim \mathfrak{e}$ from Lemma 3.12(i). So we have $V(T)-V \sim 1$ and thus $c=1$. 
Now we will consider the deep case. For $T=x+1+A$, consider Abel's Equation $V \circ T=V+1$. A formal solution is

$$
V=x+A+A \circ T+A \circ T^{[2]}+A \circ T^{[3]}+\cdots .
$$

But if $T$ is not purely deep, then $A \circ T \asymp A$ (Lemma 3.13), so series (18) does not converge. We will use the moderate version already proved (Theorem 4.1) to reduce the general case to one where $A^{\dagger} \succ 1$ (Proposition 4.2) so that $A \circ T \prec A$ and the series does converge (Proposition 4.3). But in general it cannot be grid-based (Example 4.8), so the final step works only for the well-based version of $\mathbb{T}$.

Proposition 4.2. Let $T \sim x, T>x$. There exists large positive $V$ such that $V \circ T \circ V^{[-1]}=x+1+B$ and $\mathfrak{g}^{\dagger} \succ 1$ for all $\mathfrak{g} \in \operatorname{supp} B$; that is, $x+1+B$ is purely deep. Let $0<S<x, S \succ 1$. There exists $V \in \mathcal{P}$ such that $V \circ S \circ V^{[-1]}=x-1+C$ and $\mathfrak{g}^{\dagger} \succ 1$ for all $\mathfrak{g} \in \operatorname{supp} C$.

Proof. Write $T=x\left(1+a \mathfrak{e}+A_{1}+A_{2}\right)$, where

(i) $\mathfrak{g} \prec \mathfrak{e}, \mathfrak{g}^{\dagger} \preccurlyeq 1 /(x \mathfrak{e})$ for all $\mathfrak{g} \in \operatorname{supp} A_{1}$,

(ii) $\mathfrak{g} \prec 1, \mathfrak{g}^{\dagger} \succ 1 /(x \mathfrak{e})$ for all $\mathfrak{g} \in \operatorname{supp} A_{2}$.

So $T_{1}=x\left(1+a \mathfrak{e}+A_{1}\right)$ is the moderate part of $T$ (including the shallow part), and $T-T_{1}=x A_{2}$ is the deep part of $T$. By Theorem 4.1, there is large positive $V \succ \log x$ so that $V \circ T_{1}=V+1$ and $V^{\prime} \sim 1 /(\operatorname{axe})$. So compute

$$
V \circ T-V \circ T_{1}=\int_{T_{1}}^{T} V^{\prime} \succ \int_{T_{1}}^{T} \frac{1}{x} .
$$

Now $T-T_{1}=A_{2}$, so this integral is between $A_{2} / T_{1} \sim A_{2} / x$ and $A_{2} / T \sim A_{2} / x$. So $B_{1}:=V \circ T-V \circ T_{1} \prec A_{2}$ and

$$
V \circ T=V \circ T_{1}+B_{1}=V+1+B_{1}
$$

with $B_{1}^{\dagger} \succ 1 /(x \mathfrak{e})$. So write $B=B_{1} \circ V^{[-1]}$ to get $V \circ T \circ V^{[-1]}=x+1+B$ and $B_{1}^{\dagger}=(B \circ V)^{\dagger}=\left(B^{\dagger} \circ V\right) \cdot V^{\prime}$. But $V^{\prime} \asymp 1 /(x \mathfrak{e})$ and $B_{1}^{\dagger} \succ 1 /(x \mathfrak{e})$, so $B^{\dagger} \succ 1$.

Now let $0<S<x, S \succ 1$. Then define $T:=S^{[-1]}$ to get $T>x, T \succ 1$. So as we have just seen, there is $V$ with $V \circ T \circ V^{[-1]}=x+1+B$. Take the inverse to get

$$
V \circ S \circ V^{[-1]}=\left(V \circ T \circ V^{[-1]}\right)^{[-1]}=(x+1+B)^{[-1]} .
$$

So if $(x+1+B)^{[-1]}=(x-1+C)$, we must show $C^{\dagger} \succ 1$. Now $(x+1+B) \circ(x-1+C)=$ $x$, so $x-1+C+1+B \circ(x-1+C)=x$ and therefore $C=-B \circ(x-1+C)$, so

$$
C^{\dagger}=\left(B^{\dagger} \circ(x-1+C)\right) \cdot(x-1+C)^{\prime} \succ 1 \cdot 1=1,
$$

as required.

The following proof is only for the well-based version of $\mathbb{T}$. In Example 4.8, below, we see it fails in general for the grid-based version of $\mathbb{T}$.

Proposition 4.3. $W[$ Purely Deep Abel] (a) Let $T=x+1+A, A \in \mathbb{T}, A \prec 1$, $A^{\dagger} \succ 1$. There is $V=x+B, B \in \mathbb{T}, B \prec 1, B^{\dagger} \succ 1$, such that $V \circ T=V+1$. (b) Let $T=x-1+A, A \in \mathbb{T}, A \prec 1, A^{\dagger} \succ 1$. There is $V=x+B, B \in \mathbb{T}, B \prec 1$, $B^{\dagger} \succ 1$ such that $V \circ T=V-1$. 
Proof. (a) There exist $N, M \in \mathbb{N}$ so that $\operatorname{supp} A \subset \mathfrak{G}_{N, M}$. Increase $N$ if necessary so that $N \geq M$ and $x \in \mathfrak{G}_{N, M}$. Now $\mathfrak{e}=x^{-1}$, so the deep monomials are $\mathfrak{D}=$ $\left\{\mathfrak{g} \in \mathfrak{G}_{N, M}^{\text {small }}: \mathfrak{g}^{\dagger} \succ 1\right\}$. We claim that if $B \in \mathbb{T}$, supp $B \subseteq \mathfrak{D}$, then $B(T) \prec B$ and $\operatorname{supp} B(T) \subseteq \mathfrak{D}$. Indeed, all $\mathfrak{g} \in \operatorname{supp} B$ satisfy $\mathfrak{g}(T) \prec \mathfrak{g}$ by Lemma 3.12(iv), and we may sum to conclude $B(T) \prec B$. Therefore $B(T)^{\dagger} \succcurlyeq B^{\dagger} \succ 1$. Also, $\operatorname{supp} B(T) \subseteq \mathfrak{G}_{N, M}$ by [11, Prop. 3.111].

Let $\mathcal{A}=\{x+B \in \mathbb{T}: \operatorname{supp} B \subseteq \mathfrak{D}\}$. Define $\Psi$ by $\Psi(Y):=Y \circ T-1$. We want to apply a fixed point argument to $\Psi$. First we must show that $\Psi$ maps $\mathcal{A}$ into $\mathcal{A}$. Let $x+B \in \mathcal{A}$. So $\Psi(x+B)=T+B \circ T-1=x+1+A+B(T)-1$. But $\operatorname{supp} A \subseteq \mathfrak{D}, \operatorname{supp} B \subseteq \mathfrak{D}$, so $\operatorname{supp} B(T) \subseteq \mathfrak{D}$, and thus $x+A+B(T) \in \mathcal{A}$.

Suppose $x+B_{1}, x+B_{2} \in \mathcal{A}$. Then $\operatorname{supp}\left(B_{1}-B_{2}\right) \subseteq \mathfrak{D}$ and

$$
\begin{aligned}
\Psi\left(x+B_{1}\right)-\Psi\left(x+B_{2}\right) & =\left(T+B_{1} \circ T-1\right)-\left(T+B_{2} \circ T-1\right) \\
& =\left(B_{1}-B_{2}\right) \circ T \prec B_{1}-B_{2} .
\end{aligned}
$$

So $\Psi$ is contractive.

Now we are ready to apply the well-based contraction theorem [16, Thm 4.7]. In our case where $\mathfrak{G}$ is totally ordered, the dotted ordering $\prec$ of 16 coincides with the usual ordering $\prec$. There is $V \in \mathcal{A}$ such that $\Psi(V)=V$. This is what was required.

Part (b) is proved from part (a) as before: Begin with $T=x-1+A$ purely deep, then $T^{[-1]}=x+1+A_{1}$ also purely deep; from part (a) get $V=x+B$ with $V \circ T^{[-1]}=V+1$, so compose with $T$ on the right to get $V=V \circ T+1$ as desired.

Because they depend on Proposition 4.3, the following two results are also valid only for the well-based version of $\mathbb{T}$.

Theorem 4.4. $W[$ General Abel $]$ Let $T \in \mathcal{P}$ with expo $T=0$. Then there is $V \in \mathcal{P}$ such that: (i) If $T>x$, then $V \circ T \circ V^{[-1]}=x+1$; (ii) if $T<x$, then $V \circ T \circ V^{[-1]}=x-1$.

Proof. (i) First, there is $V_{1}$ so that $T_{1}:=V_{1} \circ T \circ V_{1}^{[-1]} \sim x$. By Proposition4.2, there is $V_{2}$ so that $T_{2}:=V_{2} \circ T_{1} \circ V_{2}^{[-1]}=x+1+B$ with $B^{\dagger} \succ 1$. By Proposition 4.3 there is $V_{3}$ so that $V_{3} \circ T_{2} \circ V_{3}^{[-1]}=x+1$. Define $V=V_{3} \circ V_{2} \circ V_{1}$ to get $V \circ T \circ V^{[-1]}=x+1$.

(ii) is similar.

Corollary 4.5. $\mathrm{W}$ Let $T \in \mathcal{P}$ with expo $T=0$. Then there exists a real iteration group $\Phi(s, x)$ for $T$.

Proof. In the case $T>x$, let $V$ be as in Theorem 4.4(i); then take $\Phi(s, x)=$ $V^{[-1]} \circ(x+s) \circ V$. In the case $T<x$, let $V$ be as in Theorem 4.4(ii); then take $\Phi(s, x)=V^{[-1]} \circ(x-s) \circ V$.

Question 4.6. The proof as given here depends on the existence of inverses in $\mathcal{P}$. Is it possible to first demonstrate the solution to Abel's Equation without assuming the existence of inverses and then use that to construct inverses?

Example 4.7. Take the example $T=x+1+x e^{-x^{2}}$ of Proposition 2.6 Carrying out the iteration of Proposition 4.3. we get $V$ satisfying $V(T(x))=V(x)+1$ which 
looks like:

$$
\begin{aligned}
& \begin{array}{l}
V \\
=
\end{array} \quad x \\
& +e^{-x^{2}}\left(x+e^{-2 x}(x+1) e^{-1}+e^{-4 x}(x+2) e^{-4}+e^{-6 x}(x+3) e^{-9 x}+\cdots\right) \\
& +e^{-2 x^{2}}\left(e^{-2 x}\left(-x-4 x^{2}-2 x^{3}\right) e^{-1}+e^{-4 x}\left(x-4 x^{2}-2 x^{3}\right) e^{-4}\right. \\
& \left.\quad+e^{-6 x}\left[\left(7 x-4 x^{2}-2 x^{3}\right) e^{-9}+\left(-7-15 x-10 x^{2}-2 x^{3}\right) e^{-5}\right]+\cdots\right) \\
& +e^{-3 x^{2}}\left(e^{-2 x}\left(-x^{2}+3 x^{3}+6 x^{4}+2 x^{5}\right) e^{-1}+e^{-4 x}\left(-2 x^{2}-3 x^{3}+4 x^{4}+2 x^{5}\right) e^{-4}\right. \\
& \quad+e^{-6 x}\left[\left(+5 x^{2}-13 x^{3}+2 x^{4}+2 x^{5}\right) e^{-9}\right. \\
& \left.\left.\quad+\left(-x+38 x^{2}+74 x^{3}+44 x^{4}+8 x^{5}\right) e^{-5}\right]+\cdots\right) \\
& +e^{-4 x^{2}}\left(e^{-2 x}\left(\frac{5}{3} x^{3}+\frac{8}{3} x^{4}-4 x^{5}-\frac{16}{3} x^{6}-\frac{4}{3} x^{7}\right) e^{-1}\right. \\
& \left.\quad+e^{-4 x}\left(-x^{3}+4 x^{4}+4 x^{5}-\frac{8}{3} x^{6}-\frac{4}{3} x^{7}\right) e^{-4}+\cdots\right) \\
& +\cdots
\end{aligned}
$$

The support is a subgrid of order type $\omega^{2}$.

Of course, once we have $V$ we can compute the real iteration group $\Phi(s, x)=$ $V^{[-1]} \circ(x+s) \circ V$. For $s$ negative we get

$$
\Phi(s, x)=x+s-x e^{-(x+s)^{2}}+\cdots,
$$

so they are not contained in a common grid (or well ordered set), as noted before. But since $V$ is grid-based, all of the fractional iterates $T^{[s]}$ are also grid-based.

\section{The non-grid situation.}

Example 4.8. Here is an example where Abel's Equation has no grid-based solution:

$$
T=x+1+e^{-e^{x^{2}}} .
$$

The support for $V$, where $V \circ T=V+1$, deserves careful examination. We will use the notation:

$$
\begin{aligned}
\mathfrak{m}_{1} & =x^{-1}, \quad \mathfrak{m}_{1} \prec 1, \quad \mathfrak{m}_{1} \in \mathfrak{G}_{0}, \\
\mathfrak{m}_{2} & =e^{-x}, \quad \mathfrak{m}_{2} \prec \mathfrak{m}_{1}, \quad \mathfrak{m}_{2} \in \mathfrak{G}_{1}, \\
\mathfrak{m}_{3} & =e^{-x^{2}}, \quad \mathfrak{m}_{3} \prec \mathfrak{m}_{2}, \quad \mathfrak{m}_{3} \in \mathfrak{G}_{1}, \\
\boldsymbol{\mu} & =\left\{\mathfrak{m}_{1}, \mathfrak{m}_{2}, \mathfrak{m}_{3}\right\} \subset \mathfrak{G}_{1}, \\
L_{k} & =e^{(x+k)^{2}}=e^{k^{2}} \mathfrak{m}_{2}^{-2 k} \mathfrak{m}_{3}^{-1}, \quad k=0,1,2, \cdots, \quad \operatorname{supp} L_{k} \subset \mathfrak{J}^{\mu} \subset \mathfrak{G}_{1}, \\
\mathfrak{a}_{k} & =e^{-L_{k}}, \quad k=0,1,2, \cdots, \quad \mathfrak{a}_{k} \in \mathfrak{G}_{2}, \\
\mathfrak{b}_{k} & =x \mathfrak{m}_{2}^{-2 k} \mathfrak{m}_{3}^{-1} \mathfrak{a}_{k}, \quad \boldsymbol{\alpha}=\boldsymbol{\mu} \cup\left\{\mathfrak{b}_{k}: k=0,1,2, \cdots\right\} \subset \mathfrak{G}_{2} .
\end{aligned}
$$

Now $\boldsymbol{\alpha}$ is infinite, so it is not a ratio set in the usual sense. However, writing $\mathfrak{g}_{1} \succ \mathfrak{g}_{2}$ iff $\mathfrak{g}_{1}^{k} \succ \mathfrak{g}_{2}$ for all $k \in \mathbb{N}$, we have

$$
\mathfrak{m}_{1} \succcurlyeq \mathfrak{m}_{2} \succcurlyeq \mathfrak{m}_{3} \succcurlyeq \mathfrak{b}_{0} \succcurlyeq \mathfrak{b}_{1} \succcurlyeq \mathfrak{b}_{2} \succcurlyeq \cdots \text {. }
$$


The semigroup generated by $\boldsymbol{\alpha}$ is contained in $\mathfrak{G}_{2}$, is well ordered, and has order type $\omega^{\omega}$. Probably the solution $V$ of Abel's Equation also has support of order type $\omega^{\omega}$, but to prove it we would have to verify that many terms are not eliminated by cancellation.

Computations follow. When we write o and $\mathrm{O}$, the omitted terms all belong to $\mathfrak{G}_{2}$.

$$
\begin{aligned}
T & =x+1+\mathfrak{a}_{0}, \\
T^{2} & =x^{2}+2 x+1+2 x \mathfrak{a}_{0}+\mathrm{O}\left(\mathfrak{a}_{0}\right), \\
\mathfrak{m}_{2}^{-1} \circ T & =e^{T}=e^{x+1+\mathfrak{a}_{0}}=e \mathfrak{m}_{2}^{-1} e^{\mathfrak{a}_{0}}=e \mathfrak{m}_{2}^{-1}\left(1+\mathfrak{a}_{0}+\mathrm{o}\left(\mathfrak{a}_{0}\right)\right) \\
& =e \mathfrak{m}_{2}^{-1}+e \mathfrak{m}_{2}^{-1} \mathfrak{a}_{0}+\mathrm{o}\left(\mathfrak{m}_{2}^{-1} \mathfrak{a}_{0}\right), \\
\mathfrak{m}_{2}^{-2 k} \circ T & =e^{2 k} \mathfrak{m}_{2}^{-2 k}+2 k e^{2 k} \mathfrak{m}_{2}^{-2 k} \mathfrak{a}_{0}+\mathrm{o}\left(\mathfrak{m}_{2}^{-2 k} \mathfrak{a}_{0}\right), \\
\mathfrak{m}_{3}^{-1} \circ T & =e^{T^{2}}=e^{x^{2}+2 x+1+2 x \mathfrak{a}_{0}+\mathrm{O}\left(\mathfrak{a}_{0}\right)}=e \mathfrak{m}_{2}^{-2} \mathfrak{m}_{3}^{-1} e^{2 x \mathfrak{a}_{0}+\mathrm{O}\left(\mathfrak{a}_{0}\right)} \\
& =e \mathfrak{m}_{2}^{-2} \mathfrak{m}_{3}^{-1}\left(1+2 x \mathfrak{a}_{0}+\mathrm{O}\left(\mathfrak{a}_{0}\right)\right) \\
& =e \mathfrak{m}_{2}^{-2} \mathfrak{m}_{3}^{-1}+2 e x \mathfrak{m}_{2}^{-2} \mathfrak{m}_{3}^{-1} \mathfrak{a}_{0}+\mathrm{O}\left(\mathfrak{m}_{2}^{-2} \mathfrak{m}_{3}^{-1} \mathfrak{a}_{0}\right), \\
L_{k} \circ T & =e^{k^{2}+2 k+1} \mathfrak{m}_{2}^{-2 k-2} \mathfrak{m}_{3}^{-1}+2 e^{k^{2}+2 k+1} x \mathfrak{m}_{2}^{-2 k-2} \mathfrak{m}_{3}^{-1} \mathfrak{a}_{0}+\mathrm{O}\left(\mathfrak{m}_{2}^{-2 k-2} \mathfrak{m}_{3}^{-1} \mathfrak{a}_{0}\right), \\
\mathfrak{a}_{k} \circ T & =e^{-L_{k+1}} e^{-2 e^{(k+1)^{2}}} x \mathfrak{m}_{2}^{-2 k-2} \mathfrak{m}_{3}^{-1} \mathfrak{a}_{0}+\mathrm{O}\left(\mathfrak{m}_{2}^{-2 k-2} \mathfrak{m}_{3}^{-1} \mathfrak{a}_{0}\right) \\
& =\mathfrak{a}_{k+1}\left(1-2 e^{(k+1)^{2}} x \mathfrak{m}_{2}^{-2 k-2} \mathfrak{m}_{3}^{-1} \mathfrak{a}_{0}+\mathrm{O}\left(\mathfrak{m}_{2}^{-2 k-2} \mathfrak{m}_{3}^{-1} \mathfrak{a}_{0}\right)\right) \\
& =\mathfrak{a}_{k+1}-2 e^{(k+1)^{2}} x \mathfrak{m}_{2}^{-2 k-2} \mathfrak{m}_{3}^{-1} \mathfrak{a}_{0} \mathfrak{a}_{k+1}+\mathrm{O}\left(\mathfrak{m}_{2}^{-2 k-2} \mathfrak{m}_{3}^{-1} \mathfrak{a}_{0} \mathfrak{a}_{k+1}\right) \\
& =\mathfrak{a}_{k+1}-2 e^{(k+1)^{2}} \mathfrak{a}_{0} \mathfrak{b}_{k+1}+\mathrm{o}\left(\mathfrak{a}_{0} \mathfrak{b}_{k+1}\right), \\
\mathfrak{b}_{k} \circ T & =\left(x \mathfrak{m}_{2}^{-2 k} \mathfrak{m}_{3}^{-1} \mathfrak{a}_{k}\right) \circ T=\mathfrak{b}_{k+1}+\mathrm{o}\left(\mathfrak{b}_{k+1}\right) .
\end{aligned}
$$

The solution $V$ of Abel's Equation $V \circ T=V+1$ is

$$
V=x+1+\mathfrak{a}_{0}+\mathfrak{a}_{0} \circ T+\mathfrak{a}_{0} \circ T^{[2]}+\mathfrak{a}_{0} \circ T^{[3]}+\cdots .
$$

Without considering cancellation, we would expect that its support still has order type $\omega^{\omega}$. Even without trying to account for cancellation, we know that $\operatorname{supp} V$ contains $\left\{\mathfrak{a}_{0}, \mathfrak{a}_{1}, \mathfrak{a}_{2}, \cdots\right\}$. The logarithms $L_{k}$ are linearly independent, so the group generated by $\left\{\mathfrak{a}_{k}: k \in \mathbb{N}\right\}$ is not finitely generated, and thus $\operatorname{supp} V$ is not a subgrid.

More computation in this example yields $V^{[-1]}=x-1-\mathfrak{a}_{-1}+\cdots$ and

$$
T^{[1 / 2]}=V^{[-1]} \circ\left(x+\frac{1}{2}\right) \circ V=x+\frac{1}{2}+\mathfrak{a}_{0}-\mathfrak{a}_{1 / 2}+\cdots
$$

not grid-based. We used the notation $\mathfrak{a}_{k}=\exp \left(-\exp \left((x+k)^{2}\right)\right)$ for $k=-1$ and $1 / 2$.

Consider the proof of Proposition 4.3. How much can be done in the grid-based version? Assume grid-based $T=x+1+A, A \in \mathbb{T}, A \prec 1, A^{\dagger} \succ 1$. Write $\mathfrak{m}=\operatorname{mag} A$. Consider a ratio set $\boldsymbol{\mu}$ such that $\operatorname{supp} A \subseteq \mathfrak{m} \mathfrak{J}^{\boldsymbol{\mu}, \mathbf{0}}$. There is [13, Prop. 5.6] a "T-composition addendum" $\boldsymbol{\alpha}$ for $\boldsymbol{\mu}$ such that:

(i) if $\mathfrak{a} \in \mathfrak{J}^{\boldsymbol{\mu}, \mathbf{0}}$, then $\operatorname{supp}(\mathfrak{a} \circ T) \subseteq \mathfrak{J}^{\boldsymbol{\alpha}, \mathbf{0}}$;

(ii) if $\mathfrak{a} \prec^{\mu} \mathfrak{b}$, then $\mathfrak{a} \circ T \prec^{\alpha} \mathfrak{b} \circ T$. 
But this is not enough to carry out the contraction argument. We need a "hereditary $T$-composition addendum" $\boldsymbol{\alpha} \supseteq \boldsymbol{\mu}$ such that:

(i) if $\mathfrak{a} \in \mathfrak{J}^{\boldsymbol{\alpha}, \mathbf{0}}$, then $\operatorname{supp}(\mathfrak{a} \circ T) \subseteq \mathfrak{J}^{\boldsymbol{\alpha}, \mathbf{0}}$;

(ii) if $\mathfrak{a} \prec^{\alpha} \mathfrak{b}$, then $\mathfrak{a} \circ T \prec^{\alpha} \mathfrak{b} \circ T$.

For some deep $T$ there is such an addendum, but not for others. If there is, then a grid-based version of the contraction argument of Proposition 4.3 works. Or (for purely deep $T$ ) we can write

$$
V=x+1+A+A \circ T+A \circ T^{[2]}+A \circ T^{[3]}+\cdots
$$

with $A \succ^{\boldsymbol{\alpha}} A \circ T \succ^{\boldsymbol{\alpha}} A \circ T^{[2]} \succ^{\boldsymbol{\alpha}} \ldots$ to ensure grid-based convergence in the asymptotic topology.

For example, if $\boldsymbol{\mu}=\left\{x^{-1}, e^{-x}, e^{-x^{2}}\right\}$ and $\operatorname{supp} A \subseteq \mathfrak{J}^{\boldsymbol{\mu}, \mathbf{0}}$, then $\left\{x^{-1}, e^{-x}, x^{2} e^{-x^{2}}\right\}$ is a hereditary $T$-composition addendum. This ensures that the iteration used in Example 4.7 provides a grid-based solution $V$.

\section{UNIQUENESS}

In what sense is $T^{[s]}$ unique? This question is related to the question of commutativity for composition.

Proposition 5.1. Let $V \in \mathcal{P}$. If $V(x+1)=V+1$, then there is $c \in \mathbb{R}$ with $V=x+c$.

Proof. We claim $V^{\prime}=1$. Indeed, $V(x+1)-(x+1)=V(x)-x$, so applying Proposition 0.1 with $T=V-x$, we get $(V-x)^{\prime}=0$ or $V^{\prime}=1$. So $V=x+c$ as required.

Corollary 5.2. Let $T \in \mathcal{P}, T>x$. The solution $V \in \mathcal{P}$ of $V \circ T=V+1$ is unique up to a constant addend.

Proof. Suppose $V \circ T=V+1$ and $U \circ T=U+1$. Then $V^{[-1]} \circ(x+1) \circ V=$ $U^{[-1]} \circ(x+1) \circ U$ and $\left(U \circ V^{[-1]}\right) \circ(x+1)=(x+1) \circ\left(U \circ V^{[-1]}\right)$. By Proposition 5.1 there is $c \in \mathbb{T}$ with $U \circ V^{[-1]}=x+c$ so that $U=V+c$.

Notation 5.3. Let $T \in \mathcal{P}, s \in \mathbb{R}$. If $T>x$, define $T^{[s]}=V^{[-1]} \circ(x+s) \circ V$, where $V$ is a solution of Abel's Equation $V(T)=V+1$. If $T<x$, define $T^{[s]}=V^{[-1]} \circ(x-s) \circ V$, where $V$ is a solution of Abel's Equation $V(T)=V-1$. The transseries $T^{[s]}$ is independent of the choice of solution $V$.

Note: Even if $T$ is grid-based, it could happen that $T^{[s]}$ is not.

Proposition 5.4. Let $A, B \in \mathcal{P}, B \neq x$. If $A \circ B=B \circ A$, then there is $s \in \mathbb{R}$ with $B^{[s]}=A$.

Proof. We do the case $B>x$; the case $B<x$ is similar. Let $V \in \mathcal{P}$ solve Abel's Equation for $B$, so that $B^{[s]}=V^{[-1]} \circ(x+s) \circ V$ for $s \in \mathbb{R}$. Then $V^{[-1]} \circ(x+1) \circ$ $V \circ A=A \circ V^{[-1]} \circ(x+1) \circ V$. Compose with $V$ on the left and $V^{[-1]}$ on the right to get $(x+1) \circ\left(V \circ A \circ V^{[-1]}\right)=\left(V \circ A \circ V^{[-1]}\right) \circ(x+1)$. By Proposition 5.1, there is $s \in \mathbb{R}$ with $V \circ A \circ V^{[-1]}=x+s$. So $A=V^{[-1]} \circ(x+s) \circ V=B^{[s]}$.

If $A, B$ are grid-based, perhaps $B^{[s]}$ is in general not grid-based. But since we conclude $B^{[s]}=A$, then at least for this particular $s$ it happens to be grid-based. 
Example 5.5. Let $\theta: \mathbb{R} \rightarrow \mathbb{R}$ satisfy $\theta(1)=1$ and $\theta(s+t)=\theta(s)+\theta(t)$ for all $s, t$. By the axiom of choice, there is such a map $\theta$ other than the identity function $\theta(s)=s$. (This strange $\theta$ is everywhere discontinuous, non-measurable, and unbounded on every interval.) Let $T \in \mathcal{P}$. Then $\Phi(s, x)=T^{[\theta(s)]}$ is a real iteration group for $T$.

Here is a way to rule out such strange cases.

Proposition 5.6. Let $T \in \mathcal{P}, T>x$, and let $\Phi(s, x)$ be a real iteration group for T. Assume $\Phi(s, x)>x$ for all $s>0$. Then $\Phi(s, x)=T^{[s]}$ as in Notation [5.3.

Proof. Since $\Phi(s, x)>x$ for $s>0$, we may deduce that $s_{1}<s_{1}$ implies $\Phi\left(s_{1}, x\right)<$ $\Phi\left(s_{2}, x\right)$. Also, $\Phi(1, x)=T$, so we may deduce that $\Phi(s, x)=T^{[s]}$ for all rational $s$. Fix an irrational $s$. Since $\Phi(s, x) \circ \Phi(1, x)=\Phi(s+1, x)$, we know that $\Phi(s, x)$ commutes with $T$, so by Proposition [5.4, $\Phi(s, x)=T^{[t]}$ for some $t$. But the only $t$ satisfying $T^{\left[s_{1}\right]}<T^{[t]}<T^{\left[s_{2}\right]}$ for all rationals $s_{1}, s_{2}$ with $s_{1}<s<s_{2}$ is $t=s$ itself.

Similarly, let $T \in \mathcal{P}, T<x$, and let $\Phi(s, x)$ be a real iteration group for $T$. Assume $\Phi(s, x)<x$ for all $s>0$. Then $\Phi(s, x)=T^{[s]}$ as in Notation 5.3 .

\section{JULia EXAMPLE}

As an example we will consider fractional iterates for the function $M(x)=x^{2}+c$ near $x=+\infty$. Of course, positive integer iterates of this function are used for construction of Julia sets or the Mandelbrot set. For the theory of real transseries to be applicable, we must restrict to real values $c$. But once we have nice formulas, they can be investigated for general complex $c$. In the case $c=-2$ there is a known closed form, $M^{[s]}=2 \cosh \left(2^{s} \operatorname{acosh}(x / 2)\right)$. [Of course, $x^{2}-2=2 \cosh (2 \operatorname{acosh}(x / 2))$ is essentially the double-angle formula for cosines.] Also, of course in the case $c=0$ the closed form is $M^{[s]}=x^{2^{s}}$. For other values of $c$ no closed form is known, and it is likely that there is none (but that must be explained). [The referee provided an interesting explanation, where "having a closed form" is interpreted (as is reasonable) by "being differentiably algebraic": According to Eremenko, the integer iterates of a polynomial $M(x)$ are uniformly differentially algebraic (= satisfy the same algebraic differential equation with constant coefficients) iff $M$ is conjugate (by a linear function) to a monomial, a Chebyshev polynomial, or the negative of a Chebyshev polynomial. See 25, p. 663]. Also, one easily verifies that $M(x)=x^{2}+c$ satisfies this condition precisely if $c=0$ or $c=-2$.]

So, let $c$ be a fixed real number, and write $M(x)=x^{2}+c$. Use ratio set $\boldsymbol{\mu}=\left\{\mu_{0}, \mu_{1}, \mu_{2}, \mu_{3}\right\}$,

$$
\mu_{0}=\frac{1}{\log x}, \quad \mu_{1}=x^{-1}, \quad \mu_{2}=e^{-x}, \quad \mu_{3}=e^{-e^{x}} .
$$

Begin with $M(x)=x^{2}+c$. Then

$M_{1}:=\log \circ M \circ \exp =\log \left(e^{2 x}+c\right)=\log \left(e^{2 x}\left(1+c e^{-2 x}\right)\right)=2 x-\sum_{j=1}^{\infty} \frac{(-1)^{j} c^{j} \mu_{2}^{2 j}}{j}$. 
So $M_{1}$ is a series in powers of $\mu_{2}$. Next,

$$
\begin{aligned}
M_{2}:=\log \circ M_{1} \circ \exp & =\log \left(2 e^{x}-\sum_{j=1}^{\infty} \frac{(-1)^{j} c^{j} \mu_{3}^{2 j}}{j}\right) \\
& =\log \left(2 e^{x}\left(1-\sum_{j=1}^{\infty} \frac{(-1)^{j} c^{j} \mu_{2} \mu_{3}^{2 j}}{2 j}\right)\right) .
\end{aligned}
$$

Writing $A$ for the series (in powers of $\mu_{2}, \mu_{3}$ ),

$$
M_{2}=x+\log 2-\sum_{j=1}^{\infty} \frac{A^{j}}{j}=x+\log 2+\frac{c}{2} \mu_{2} \mu_{3}^{2}-\frac{c^{2}}{4} \mu_{2} \mu_{3}^{4}-\frac{c^{2}}{8} \mu_{2}^{2} \mu_{3}^{4}+\mathrm{O}\left(\mu_{2} \mu_{3}^{6}\right) .
$$

The $\mathrm{O}$ term represents $\mu_{2} \mu_{3}^{6}$ times a series in $\mu_{2}, \mu_{3}$ with non-negative exponents.

Note that $M_{2}$ is deep in the sense of Definition 3.2. so the iterates will be computed using Abel's Equation (use of $\log 2$ instead of 1 gives us simpler coefficients). The solution $V$ of $V \circ M_{2}=V+\log 2$ is found by iteration: $V_{0}=x$, $V_{n+1}=V_{n} \circ M_{2}-\log 2$. The result is

$$
V=x+\frac{c}{2} \mu_{2} \mu_{3}^{2}+\frac{c-c^{2}}{4} \mu_{2} \mu_{3}^{4}-\frac{c^{2}}{8} \mu_{2}^{2} \mu_{3}^{4}-\frac{c^{2}}{2} \mu_{2} \mu_{3}^{6}-\frac{c^{2}}{8} \mu_{2}^{2} \mu_{3}^{6}+\mathrm{O}\left(\mu_{2} \mu_{3}^{8}\right) .
$$

The $\mathrm{O}$ is a series in $\mu_{2}, \mu_{3}$. For $c=-2$, the closed form is

$$
V=\log \operatorname{acosh} \frac{1}{2} e^{e^{x}}
$$

The inverse is computed as in [12, Prop. 4.19]:

$$
\begin{array}{r}
V^{[-1]}=x-\frac{c}{2} \mu_{2} \mu_{3}^{2}+\frac{c-c^{2}}{4} \mu_{2} \mu_{3}^{4}-\frac{c^{2}}{8} \mu_{2}^{2} \mu_{3}^{4}+\frac{c^{3}-3 c^{2}}{6} \mu_{2} \mu_{3}^{6} \\
+\frac{c^{3}-c^{2}}{8} \mu_{2}^{2} \mu_{3}^{6}+\frac{c^{3}}{24} \mu_{2}^{3} \mu_{3}^{6}+\mathrm{O}\left(\mu_{2} \mu_{3}^{8}\right) .
\end{array}
$$

The iteration group is then a computation. For any real $s$,

$$
M_{2}^{[s]}=V^{[-1]}(V(x)+s \log 2) .
$$

For the moment we fix an $s$ and augment our ratio set with

$$
\mu_{4}=x^{-2^{s}}, \quad \mu_{5}=e^{-2^{s} x}, \quad \mu_{6}=e^{-2^{s} e^{x}} .
$$

Then

$$
\begin{aligned}
M_{2}^{[s]}=x+s \log 2 & +\frac{c}{2} \mu_{2} \mu_{3}^{2}-2^{-1-s} c \mu_{2} \mu_{6}^{2}+\frac{c-c^{2}}{4} \mu_{2} \mu_{3}^{4}-\frac{c^{2}}{8} \mu_{2}^{2} \mu_{3}^{4} \\
& +\frac{c^{2}}{2} \mu_{2} \mu_{3}^{2} \mu_{6}^{2}+2^{-2-s} c^{2} \mu_{2}^{2} \mu_{3}^{2} \mu_{6}^{2}+\mathrm{O}\left(\mu_{2} \mu_{3}^{6}+\mu_{2} \mu_{6}^{4}\right) .
\end{aligned}
$$

Which of the two terms in the $\mathrm{O}$ is larger depends on the value of $s$. The relative sizes of the terms exhibited also depend on the value of $s$. If $s>0$, then $\mu_{3} \succ \mu_{6}$, so $M_{2}^{[s]}=x+s \log 2+(c / 2) \mu_{2} \mu_{3}^{2}+\cdots$. If $s<0$, then $\mu_{3} \prec \mu_{6}$, so $M_{2}^{[s]}=$ $x+s \log 2-2^{-1-s} c \mu_{2} \mu_{6}^{2}+\cdots$. But even within these cases, the relative sizes of the remaining terms vary as $s$ varies. 
Continue:

$$
\begin{aligned}
M_{1}^{[s]} & =\exp \circ M_{2}^{[s]} \circ \log =2^{s} x+2^{-1+s} c \mu_{2}^{2}-\frac{c}{2} \mu_{5}^{2}+2^{-2+s}\left(c-c^{2}\right) \mu_{2}^{4} \\
& +2^{-1+s} c^{2} \mu_{2}^{2} \mu_{5}^{2}+\left(2^{-2+s}\left(c^{2}-c^{3}\right)-2^{-2+2 s} c^{3}\right) \mu_{2}^{4} \mu_{5}^{2}-\frac{c^{2}+c}{4} \mu_{5}^{4}+\mathrm{O}\left(\mu_{2}^{6}+\mu_{5}^{6}\right) .
\end{aligned}
$$

This is a series in $\mu_{1}, \mu_{2}, \mu_{5}$. The coefficients involve rational numbers and powers of $2^{s}$. We do not know if $\mu_{1}$ actually appears: up to this point, all terms with $\mu_{1}$ cancel. Next,

$$
\begin{aligned}
M^{[s]}= & \exp \circ M_{1}^{[s]} \circ \log =x^{2^{s}}\left(1+2^{-1+s} c \mu_{1}^{2}-\frac{c}{2} \mu_{4}^{2}\right. \\
& \left.+\left(2^{-2+s}\left(c-c^{2}\right)+2^{-3+2 s} c^{2}\right) \mu_{1}^{4}+2^{-2+s} c^{2} \mu_{1}^{2} \mu_{4}^{2}+\mathrm{O}\left(\mu_{1}^{6}+\mu_{4}^{4}\right)\right) .
\end{aligned}
$$

This is a series in $\mu_{0}, \mu_{1}, \mu_{4}$, but we do not know if $\mu_{0}=1 / \log x$ actually appears. As before, the relative size of the terms depends on the value of $s$.

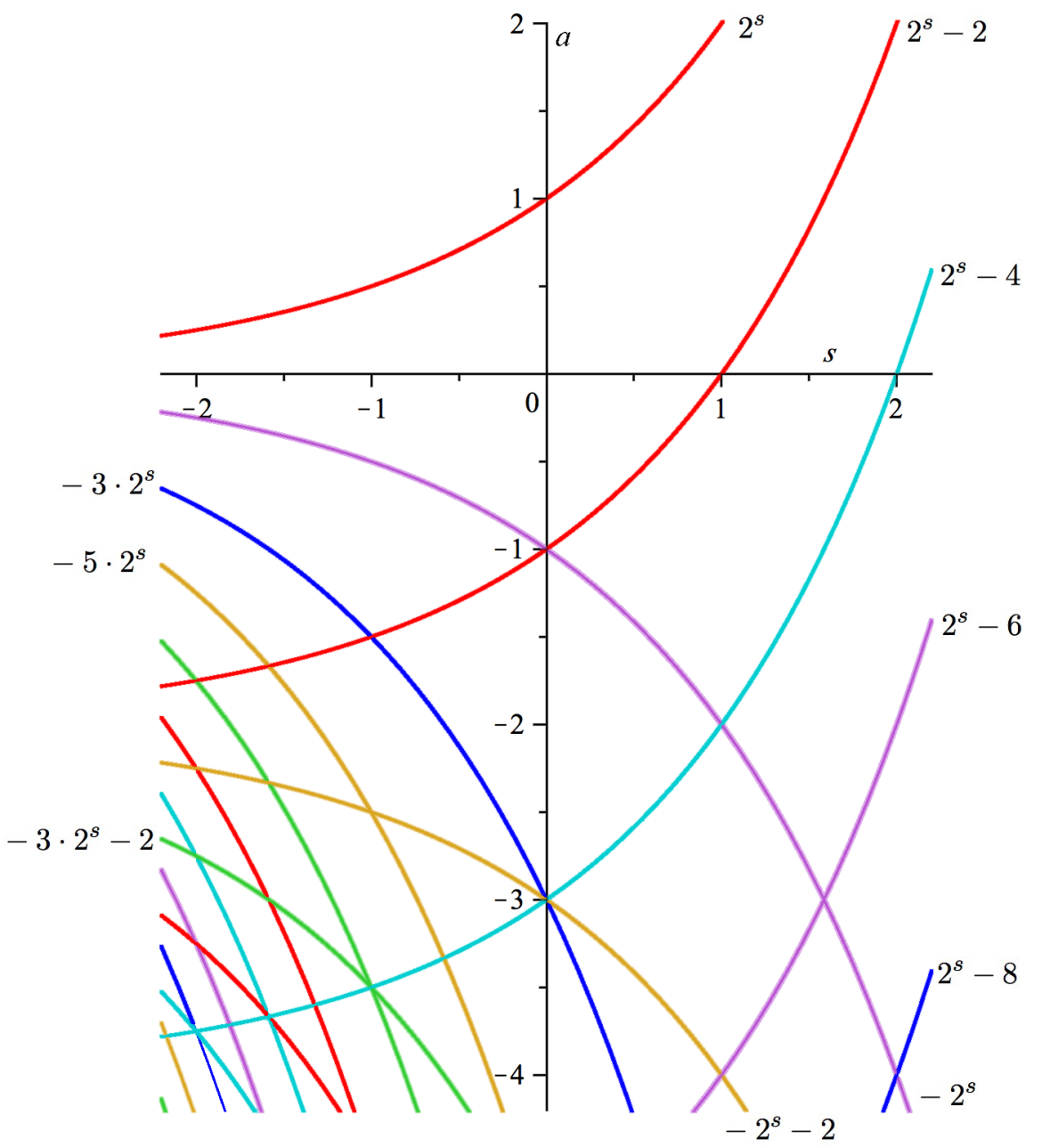

Figure 1. Monomials $x^{a}$ that support $M^{[s]}$, where $M=x^{2}+c$ 
Figure 1 illustrates the support of $M^{[s]}$ depending on $s$. The support of $M^{[s]}$ consists of certain monomials of the form $x^{a}$, where points $(s, a)$ are shown in the figure. We have assumed that logarithmic factors are, indeed, missing. Or perhaps we could say: any monomials with logarithmic factors differ only infinitesimally from the terms shown, so even if they do exist, they make no difference in the picture.

Let us substitute a few example values of $s$ into (19):

$$
\begin{aligned}
& M^{[1]}=x^{2}\left(1+c x^{-2}+\mathrm{O}\left(x^{-6}\right)\right)=x^{2}+c+\mathrm{O}\left(x^{-4}\right) \\
& M^{[-1]}=x^{1 / 2}\left(1-\frac{c}{2} x^{-1}+\mathrm{O}\left(x^{-2}\right)\right)=x^{1 / 2}-\frac{c}{2} x^{-1 / 2}+\mathrm{O}\left(x^{-3 / 2}\right), \\
& M^{[1 / 2]}=x^{\sqrt{2}}+2^{-1 / 2} c x^{\sqrt{2}-2}-\frac{c}{2} x^{-\sqrt{2}}+\left(2^{-3 / 2}\left(c-c^{2}\right)+\frac{c^{2}}{4}\right) x^{\sqrt{2}-4} \\
&+2^{-3 / 2} c^{2} x^{-2-\sqrt{2}}+\mathrm{O}\left(x^{-3 \sqrt{2}}\right) .
\end{aligned}
$$

In case $c=-2$ we have

$$
M^{[1 / 2]}=x^{\sqrt{2}}-\sqrt{2} x^{\sqrt{2}-2}+x^{-\sqrt{2}}+\left(1-\frac{3}{\sqrt{2}}\right) x^{\sqrt{2}-4}+\sqrt{2} x^{-2-\sqrt{2}}+\mathrm{O}\left(x^{-3 \sqrt{2}}\right),
$$

which does match the transseries for the closed form

$$
M^{[1 / 2]}=2 \cosh \left(\sqrt{2} \operatorname{acosh} \frac{x}{2}\right) .
$$

Problem. Of course the glaring loose end that remains is a reasonably simple proof for Proposition 0.5 .

\section{REFERENCES}

1. M. Aschenbrenner, L. van den Dries, Asymptotic differential algebra. In [8], pp. 49-85 MR2130825 (2006c:12007)

2. I. N. Baker, Zusammensetzungen ganzer Funktionen. Math. Z. 69 (1958) 121-163 MR.0097532 $(20: 4000)$

3. I. N. Baker, Permutable power series and regular iteration. J. Austral. Math. Soc. 2 (1961) 265-294 MR0140666 (25:4082)

4. A. Cayley, On some numerical expansions. Quarterly Journal of Pure and Applied Mathematics 3 (1860) 366-369. Also in: Collected Works vol. IV, pp. 470-472

5. P. M. Cohn, Universal Algebra. Harper \& Row, New York, 1965 MR0175948 (31:224)

6. O. Costin, Topological construction of transseries and introduction to generalized Borel summability. In [8], pp. 137-175 MR2130829 (2006e:40009)

7. O. Costin, Global reconstruction of analytic functions from local expansions and a new general method of converting sums into integrals. preprint, 2007. http://arxiv.org/abs/math/0612121

8. O. Costin, M. D. Kruskal, A. Macintyre (eds.), Analyzable Functions and Applications (Contemp. Math. 373). Amer. Math. Soc., Providence RI, 2005 MR2130822 (2005j:00012)

9. L. van den Dries, A. Macintyre, D. Marker, Logarithmic-exponential series. Annals of Pure and Applied Logic 111 (2001) 61-113 MR.1848569 (2002i:12007)

10. J. Écalle, Nature du groupe des ordres d'itération complexes d'une transformation holomorphe au voisinage d'un point fixe de multiplicateur 1. C. R. Acad. Sci. Paris Sér. A-B 276 (1973) A261-A263 MR0340569 (49:5321)

11. G. Edgar, Transseries for beginners. Real Analysis Exchange 35 (2010) 253-310 MR2683600 (2011m:41058)

12. G. Edgar Transseries: composition, recursion, and convergence. forthcoming http://arxiv.org/abs/0909.1259v1 or http://www.math.ohio-state.edu/ edgar/preprints/trans_compo/ 
13. G. Edgar, Transseries: ratios, grids, and witnesses. forthcoming http://arxiv.org/abs/0909.2430v1 or http://www.math.ohio-state.edu/ edgar/preprints/trans_wit/

14. G. Edgar, Tetration in transseries. forthcoming

15. P. Erdös, E. Jabotinsky, On analytic iteration. J. Analyse Math. 8 (1960) 361-376 MR.0125943 (23:A3240)

16. J. van der Hoeven, Operators on generalized power series. Illinois J. Math. 45 (2001) 11611190 MR.1894891 (2003c:16058)

17. J. van der Hoeven, Transseries and Real Differential Algebra (Lecture Notes in Mathematics 1888). Springer, New York, 2006 MR2262194 (2007f:34003)

18. J. van der Hoeven, Transserial Hardy fields. Astérisque 323 (2009) 453-487 MR 2647983 (2011i:12011)

19. A. Korkine, Sur un problème d'interpolation. Bulletin des Sciences Mathématiques et Astronomiques (2) 6 (1882) 228-242

20. M. Kuczma, Functional Equations in a Single Variable. Monografie Matematyczne 46, Państwowe Wydawnictwo Naukowe, Warsaw, 1968 MR0228862 (37:4441)

21. M. Kuczma, B. Choczewski, G. Roman, Iterative Functional Equations. Encyclopedia of Mathematics and Its Applications 32, Cambridge University Press, Cambridge, 1990 MR:1067720 (92f:39002)

22. S. Kuhlmann, Ordered Exponential Fields. American Mathematical Society, Providence, RI, 2000 MR $1760173(2002 \mathrm{~m}: 12004)$

23. L. S. O. Liverpool, Fractional iteration near a fix point of multiplier 1. J. London Math. Soc. 9 (1974) 599-609 MR0364611 (51:865)

24. M. Rosenlicht, Growth properties of functions in Hardy fields. Trans. Amer. Math. Soc. 299 (1987) 261-272 MR869411 (88b:12010)

25. L. Rubel, Some research problems about algebraic differential equations, II. Illinois J. Math. 36 (1992) 659-680 MR:1215800 (94c:34003)

26. H. F. Wilf, Generatingfunctionology. Academic Press, Boston, 1990. http://www. math. upenn.edu/ wilf/DownldGF.html

Department of Mathematics, The Ohio State University, 231 West Eighteenth AvEnue, Columbus, OHio 43210

E-mail address: edgar@math.ohio-state.edu 